\title{
Free Boundary Problems for Parabolic Equations II. Evaporation or Con- densation of a Liquid Drop
}

\section{AVNER FRIEDMAN}

\author{
Communicated by H. LEwY
}

\section{Table of Contents}

Introduction . . . . . . . . . . . . . . 19

1. Statement of the existence theorem . . . . . . . . . . . 21

2. The integral equation . . . . . . . . . . . . . 23

3. Existence and uniqueness for small times . . . . . . . . . 25

4. Existence and uniqueness for all times in case of condensation . . . . 33

5. An a priori bound on $s(t)$ for $\alpha<0 \ldots \ldots$. . . . . . . 35

6. Existence and uniqueness for all times in case of evaporation . . . . 38

7. Further $a$ priori bounds . . . . . . . . . . . . . . . . 40

8. Continuity and analyticity of the solution with respect to $\alpha$. . . . 42

9. Asymptotic behavior of solutions for $\alpha<0$ as $t \rightarrow \infty \ldots \ldots$. . . 54

References .............. . 66

Introduction. We shall refer to [2] as Part I. In Part I we proved the existence and uniqueness of the solution of Stefan problems for all future time. In this Part II we shall discuss problems of condensation and evaporation of one liquid drop surrounded by vapor of its own substance. We proceed to describe the main results.

If the initial vapor density on the surface of the drop is equal to the saturation density, then in case of condensation $(\alpha<0)$ existence and uniqueness are asserted for all future times and in case of evaporation $(\alpha>0)$ as long as the radius $s(t)$ of the drop remains positive. Here $\alpha$ is a certain physical quantity which in case $\alpha<0$ is assumed to be sufficiently small, and in case $\alpha>0$ is assumed to be $<1$. If the initial vapor density on the surface of the drop is not

Prepared under ONR Contract Nonr-222(37) (NR 041 157) with the University of California in Berkeley. 
equal to the saturation density, then existence is asserted in both cases ( $\alpha \gtrless 0$ ) as above, provided $|\alpha|$ is sufficiently small, but uniqueness is asserted only under the additional assumption that the drop does not start to grow "too fast". It is also asserted that, in case $\alpha<0, s(t) / t^{\frac{1}{2}}$ remains bounded from above as $t \rightarrow \infty$.

The proof of the above results is given in $\$ \$ 2-6$. In $\$ 2$ the problem is reduced to solving a nonlinear integral equation. This equation differs from the analogous equation of Part $I$ in that it contains terms which have a singularity like $t^{-\frac{1}{2}}$ at $t=0$. In $\S 3$ the equation is solved for a small interval of time, the length depending on $\sup _{x}|u(x, t)|$, not on $\sup _{x}\left|u_{x}(x, t)\right|$ as in Part I. In $\$ 4$ we continue the solution step by step to all future times in the case $\alpha<0$. In $\$ 5$ we prove the above mentioned a priori bound on $s(t)$. Finally, in $\$ 6$ we continue the solution into the future in the case $\alpha>0$, using a method based on ideas of Part I, $\$ 6$ and finding an $a$ priori bound on $u_{x}(x, t)$.

In $\$ 7$ we prove some $a$ priori bounds on $s(t)$ under special assumptions on the initial density. It is also proved that in case $\alpha>0$ the drop disappears after a finite time $t_{0}$. As $\alpha \rightarrow 0,2 \alpha t_{0} \rightarrow b^{2}$ where $b=s(0)$.

In $\$ 8$ we prove that in every interval of time $\sigma, 0<\sigma<\infty$, the solution is analytic in the parameter $\alpha$, provided $|\alpha| \sigma$ is smaller than some constant depending only on $b$ and on the initial vapor density. The proof consists of two steps: (i) We prove that the solution of the nonlinear integral equation mentioned above is analytic in $\alpha$. The method follows the classical procedure of extending the integral equation to the case of complex $\alpha$ and proving that the solution exists and has complex $\alpha$-derivative. From the analyticity of the solution follows the analyticity of $s(t) \equiv s(t, \alpha)$ with respect to $\alpha$. (ii) Performing the transformation $y=x-s(t, \alpha)$ which maps the free boundary $x=s(t, \alpha)$ onto the $y$-axis and defining $U(y, t)=u(x, t)$, we obtain for $U$ a differential system depending on $\alpha$. Substituting $s=\sum s_{n} \alpha^{n}, U=\sum U_{n} \alpha^{n}$ in this system, we obtain recursive formulas for the $U_{n}$. We then prove the existence of the $U_{n}$, step by step, and the convergence of $\sum U_{n} \alpha^{n}$ and some of its derivatives.

In $\$ 9$ we prove that if $\alpha<0,|\alpha|$ is small and $t$ is sufficiently large, then

$$
s(t, \alpha) \sim(2|\alpha| t)^{\frac{1}{2}}, \quad \dot{s}(t, \alpha) \sim\left(\frac{|\alpha|}{2 t}\right)^{\frac{1}{2}} .
$$

These results are used to find (within an error term) a simple asymptotic expression for $u(x, t, \alpha)$ depending on $x / t^{\frac{1}{2}}$. In particular, it follows that (asymptotically) the vapor density is a linear function of the ratio of the radius of the drop to the distance from its center.

As mentioned in the introduction to Part I, Kolodner [6] developed a method, entirely different from ours, to study some free boundary problems. Using this method, Keller, Kolodner \& Ritger [3], Kolodner [4], [5] and Kolodner \& RITGER [7] studied problems of evaporation and condensation, but only in the special case that the initial vapor density is constant. In [4], [5], [7] existence and uniqueness theorems were proved. 
The results of this Part II can be extended to general parabolic equations in one space-dimension with smooth coefficients.

In Part III of this work, to appear in this journal, we shall study the problems of one gas bubble surrounded by liquid of its own substance.

During the preparation of this paper the author had stimulating discussions with Hans Lewr, and he wishes to extend his thanks to him.

\section{Statement of the Existence Theorem}

A liquid in the presence of an undersaturated mixture containing its own vapor will evaporate, while if the mixture is supersaturated the vapor will condense. Assume that we have one 3-dimensional drop, that in the process of evaporation or condensation the drop will remain spherical and that the saturation density $g$ is independent of the radius of the drop. Assume also that in the case of condensation no new drops are created. Let the constant $\rho$ denote the density of the drop. We further assume that the initial density of the vapor is a function $c_{0}(x)$ of $x$, where $x$ denotes the distance from the center of the drop, and that $c_{0}=\lim c_{0}(x)(x \rightarrow \infty)$ exists and is different from $g$.

Denoting by $D$ the coefficient of diffusion and making the substitutions

$$
x \rightarrow x, \quad t \rightarrow \frac{t}{D}, \quad c(x, t) \rightarrow z(x, t)=\frac{c(x, t)-c_{0}}{g-c_{0}}
$$

where $c(x, t)$ is the vapor density at the point $(x, t)$, we get for $z(x, t)$ the following system of equations (see also [6]):

$$
\begin{gathered}
\Delta z=z_{t} \text { for } s(t)<x<\infty, \quad t>0 \\
z(x, 0)=\psi \\
\psi(x) \text { for } x>s(0)=b \quad(b>0), \\
z(s(t), t)=1 \text { for } t>0 \\
\alpha z_{x}(s(t), t)=\dot{s}(t) \text { for } t>0
\end{gathered}
$$

where $s(t)$ is the radius of the drop and

$$
\alpha=\frac{g-c_{0}}{\rho-g}, \quad \psi(x)=\frac{c_{0}(x)-c_{0}}{g-c_{0}} .
$$

In what follows we impose on the solution $z(x, t)$ the following conditions at infinity:

$$
\begin{gathered}
x z(x, t) \text { and }(\partial / \partial x)[x z(x, t)] \text { are bounded as } x \rightarrow \infty, \\
\text { uniformly with respect to } t \text { in finite intervals. }
\end{gathered}
$$

Thus we shall have to assume that $x \psi(x)$ and $(\partial / \partial x)[x \psi(x)]$ satisfy the same boundedness conditions. This means that $c_{0}(x)$ tends to its limit $c_{0}$ "sufficiently fast". We shall also assume below that $\psi(x) \leqq 1$. This means that

$$
\text { if } \alpha>0 \text { (or } c_{0}<g \text { ) then } c_{0}(x) \leqq g,
$$


and similarly

$$
\text { if } \alpha<0 \quad \text { (or } c_{0}>g \text { ) then } c_{0}(x) \geqq g \text {. }
$$

(Note that if $\alpha<0$ then necessarily $\rho>c_{0}$, hence $\alpha>-1$.) The first case is that of a totally undersaturated mixture and the second case corresponds to a totally supersaturated mixture.

Using the maximum principle [8; Theorem 2] we easily conclude that $z_{x}(s(t), t)$ $\leqq 0$. Therefore, by (1.4), if $\alpha>0$ then $\dot{s}(t) \leqq 0$ and the drop decreases (evaporation), while if $\alpha<0$ then $\dot{s}(t) \geqq 0$ and the drop grows (condensation). As in Part I we can prove that if $\psi(x) \equiv 1$ then $s(t)$ is strictly monotone in $t$.

Denoting $u(x, t)=x z(x, t)$, the system (1.1)-(1.5) takes the form

$$
\begin{gathered}
u_{x x}=u_{t} \text { for } s(t)<x<\infty, \quad t>0, \\
u(x, 0)=\varphi(x) \text { for } b<x<\infty \quad(\varphi(b)=s(0) \psi(b) \leqq s(0)), \\
u(s(t), t)=s(t) \text { for } t>0, \\
\alpha u_{x}(s(t), t)=s(t) \dot{s}(t)+\alpha \text { for } t>0, \\
u(x, t) \text { and } u_{x}(x, t) \text { are bounded as } x \rightarrow \infty, \\
\text { uniformly with respect to } t \text { in finite intervals. }
\end{gathered}
$$

Definition. A pair of functions $u(x, t), s(t)$ is called a solution of the system (1.6)-(1.10) for $t<\sigma(\sigma \leqq \infty)$ if (i) $s(t)(0 \leqq t<\sigma)$ is a continuous function and $s(0)=b$; (ii) $\dot{s}(t)(0<t<\sigma)$ is a continuous function and $\lim t^{\frac{1}{2}} \dot{s}(t)$ exists (or $\lim t^{\frac{1}{2}} u_{x}(s(t), t)$ exists) as $t \rightarrow 0$; (iii) $u_{x x}$ and $u_{t}$ are continuously differentiable for $s(t)<x<\infty, 0<t<\sigma$; (iv) $u_{x}$ is continuously differentiable for $s(t) \leqq$ $x<\infty, 0<t<\sigma$; (v) $u$ is continuous for $s(t) \leqq x<\infty, 0<t<\sigma$ and $s(0)<$ $x<\infty, t=0$ and $\varphi(b) \leqq \lim \inf u \leqq \lim \sup u \leqq s(0)$ as $(x, t) \rightarrow(b, 0)$ and, finally, (vi) $u(x, t)$ and $s(t)$ satisfy the equations (1.6)-(1.10).

If $\varphi(b)=b$ then we shall also demand that $\dot{s}(t)$ is continuous at $t=0$ and that $u(x, t)$ is continuous at $(b, 0)$.

We shall need the following assumption on $\varphi(x)$ :

( $\Phi)$ The functions $\varphi(x), \dot{\varphi}(x)$ are continuous and bounded for $b \leqq x<\infty$ and $\int_{b}^{\infty}|\varphi(x)| d x$ exists.

Throughout this paper there will appear various constants which depend on $b, \alpha$ and $\varphi(x)$. Unless the contrary is explicitly stated, these constants actually depend only on a lower bound on $b$, an upper bound on $|\alpha|$ and an upper bound on the quantities

$$
\underset{b<x<\infty}{\operatorname{l.u.b} .}|\varphi(x)|, \quad \underset{b<x<\infty}{\text { l.u.b. }}|\dot{\varphi}(x)|, \quad \int_{b}^{\infty}|\varphi(x)| d x .
$$

Theorem 2. Assume that $\varphi(x)$ satisfies the condition ( $\Phi)$. (i) If $\varphi(b)=b$ and $\alpha<0$ and satisfies (3.49), then there exists a unique solution $u(x, t), s(t)$ of the 
system (1.6)-(1.10) defined for all $0 \leqq t<\infty$. Furthermore,

$$
s(t) \leqq A+B t^{\frac{1}{2}} \quad(0 \leqq t<\infty)
$$

where $A, B$ are constants depending only on $\alpha, b, \varphi(x)$. (ii) If $\varphi(b)=b$ and $0<\alpha<1$, then there exists a solution $u(x, t), s(t)$ as long as $s(t)$ remains positive. The solution is uniquely determined. (iii) If $\varphi(b)<b$ then the results of (i), (ii) about existence remain true provided that in (ii) we also assume that $\alpha$ satisfies (3.49). The solution is uniquely determined by the additional condition (3.54) (with $s_{0}$ replaced by $s$ ).

Remark. In $\$ 7$ it will be proved that if $0<\alpha<1$ there exists $t_{0}<\infty$ such that $s(t) \rightarrow 0$ as $t \rightarrow t_{0}$, provided $\varphi(x)$ satisfies $(\Phi)$ and provided $\dot{\varphi}(x) \rightarrow 0$ as $x \rightarrow \infty$. It can also be proved that if $\alpha<0, s(t) \geqq A^{\prime} t^{\frac{1}{2}}-B^{\prime}$ where $A^{\prime}, B^{\prime}$ are positive constants depending on $\alpha, b, \varphi(x)$.

The proof of Theorem 2 is given in $\$ \$ 2-6$. In $\$ 2$ we transform the problem into a problem of solving a nonlinear integral equation. In $\$ 3$ we solve the integral equation for small intervals of time by a method different in some important respects from the method of Part I. In $\$ 4$ we repeat step by step the process of $\S 3$ and prove that this process can be continued so as to obtain the assertions of the theorem about existence and uniqueness in case $\alpha<0$. In $\$ 5$ we prove (1.11). Finally, in $\$ 6$ we complete the proof of Theorem 2 in case $\alpha>0$.

\section{The Integral Equation}

Suppose $u(x, t), s(t)$ form a solution of the system (1.6)-(1.10). Using Green's identity with $u(\xi, \tau)$ and

$$
K(x, t ; \xi, \tau)=\frac{1}{2 \pi^{\frac{1}{2}}(t-\tau)^{\frac{1}{2}}} \exp \left\{-\frac{(x-\xi)^{2}}{4(t-\tau)}\right\}
$$

(as a function of $(\xi, \tau)$ ) in the domain $s(\tau)<\xi<\infty, \epsilon<\tau<t-\epsilon$ and using (1.6), (1.7), (1.8), (1.9), (1.10), then letting $\epsilon \rightarrow 0$, we obtain, for $s(t)<x<\infty$, $t>0$,

$$
\begin{aligned}
u(x, t)=\int_{b}^{\infty} K(x, t ; \xi, 0) \varphi(\xi) & d \xi+\alpha \int_{0}^{t} K(x, t ; s(\tau), \tau) d \tau \\
& -\int_{0}^{t} K_{x}(x, t ; s(\tau), \tau) s(\tau) d \tau \\
& -(1+\alpha) \int_{0}^{t} K(x, t ; s(\tau), \tau) u_{x}(s(\tau), \tau) d \tau
\end{aligned}
$$

Denote

$$
v(t)=u_{x}(s(t), t)
$$


The $x$-derivative of the third term on the right side of (2.2) is equal to

$$
\begin{aligned}
-\int_{0}^{t} & K_{t}(x, t ; s(\tau), \tau) s(\tau) d \tau=\int_{0}^{t}\left[\frac{d}{d \tau} K(x, t ; s(\tau), \tau)\right] s(\tau) d \tau \\
& -\int_{0}^{t} K_{\xi}(x, t ; s(\tau), \tau) \dot{s}(\tau) s(\tau) d \tau=-K(x, t ; b, 0) b \\
& -\int_{0}^{t} K(x, t ; s(\tau), \tau) \dot{s}(\tau) d \tau-\int_{0}^{t} K_{\xi}(x, t ; s(\tau), \tau)(\alpha v(\tau)-\alpha) d \tau
\end{aligned}
$$

where we made use of (1.9). Note that if in Lemma 1, Part I, we let $x \rightarrow s(t)+0$, then the jump would be $-\frac{1}{2} \rho(t)$ (instead of $\frac{1}{2} \rho(t)$ ). Differentiating (2.2) with respect to $x$ and making use of (2.4) and (1.9) we obtain, on letting $x \rightarrow s(t)+0$ and using the last remark,

$$
\begin{aligned}
v(t)= & -2 b K(s(t), t ; b, 0)+2 \int_{b}^{\infty} K_{x}(s(t), t ; \xi, 0) \varphi(\xi) d \xi \\
& +2 \alpha \int_{0}^{t} K(s(t), t ; s(\tau), \tau) \frac{d \tau}{s(\tau)}-2 \alpha \int_{0}^{t} K(s(t), t ; s(\tau), \tau) \frac{v(\tau)}{s(\tau)} d \tau \\
& -2 \int_{0}^{t} K_{x}(s(t), t ; s(\tau), \tau) v(\tau) d \tau
\end{aligned}
$$

If we integrate (1.9) and make use of (2.3) we find that

$$
s(t)=\left[b^{2}-2 \alpha\left(t-\int_{0}^{t} v(\tau) d \tau\right)\right]^{\frac{1}{2}} .
$$

We have thus proved that if $u(x, t), s(t)$ form a solution of the system (1.6)(1.10) for $0 \leqq t<\sigma$, then $v(t)$ (defined by (2.3)) is a solution of the nonlinear integral equation (2.5) for $0<t<\sigma$ (with $s(t)$ defined by (2.6)). Recall that the definition of "solution" implies that $t^{\frac{1}{2}} v(t)$ is a continuous function for $0 \leqq t<\sigma$. Conversely (as in Part I) one can show that if $v(t)$ is a solution of (2.5) (with $s(t)$ defined by (2.6), for $0<t<\sigma$, and if $s(t)>0$ and $t^{\frac{1}{2}} v(t)$ is continuous for $0 \leqq t<\sigma$, then $u(x, t), s(t)$ form a solution of (1.6)-(1.10) where $u$ is defined by (2.2) with $u_{x}(s(t), t)$ replaced by $v(t)$.

There are two reasons why we did not try to eliminate the singularity (of order $t^{-\frac{1}{2}}$ as $t \rightarrow 0$ ) of the second term on the right side of (2.5) by performing integration by parts. First, in case $\varphi(b) \neq b$ we would, after integration by parts, still get a term

$$
-2(b-\varphi(b)) K(s(t), t ; b, 0)
$$

which is singular as $t \rightarrow 0$. The second reason is that we wish to establish $a$ priori limitations depending on $\|\varphi\|$ and not on $\|\dot{\varphi}\|$. For the method of Part I of finding an a priori bound on $v(t)$ or $u_{x}(x, t)$ fails in the present situation if $\alpha<0$. 
Since the first two terms on the right side of (2.5) have a singularity at $t=0$ which behaves essentially like $t^{-\frac{3}{3}}$, we define

$$
\bar{v}(t)=t^{\frac{1}{2}} v(t)
$$

and transform the integral equation (2.5) into an integral equation in $\bar{v}(t)$.

\section{Existence and Uniqueness for Small Times}

Consider the transformation $\bar{w}=\bar{T} \bar{v}$ defined as follows:

$$
\begin{aligned}
& \bar{w}(t)=-2 b t^{\frac{1}{2}} K(s(t), t ; b, 0)+2 \int_{b}^{\infty} t^{\frac{1}{2}} K_{x}(s(t), t ; \xi, 0) \varphi(\xi) d \xi \\
& \quad+2 \alpha \int_{0}^{t} t^{\frac{1}{2}} K(s(t), t ; s(\tau), \tau) \frac{d \tau}{s(\tau)}-2 \alpha \int_{0}^{t} t^{\frac{1}{2}} K(s(t), t ; s(\tau), \tau) \frac{\bar{v}(\tau)}{\tau^{\frac{1}{2}} s(\tau)} d \tau \\
& \quad-2 \int_{0}^{t} t^{\frac{1}{2}} K_{x}(s(t), t ; s(\tau), \tau) \frac{\bar{v}(\tau)}{\tau^{\frac{1}{2}}} d \tau
\end{aligned}
$$

where

$$
s(t)=\left[b^{2}-2 \alpha\left(t-\int_{0}^{t} \frac{\bar{v}(\tau)}{\tau^{\frac{1}{2}}} d \tau\right)\right]^{\frac{1}{2}} .
$$

This transformation is defined on the Banach space $C_{\sigma}$ of functions $\bar{v}(t)$ continuous for $0 \leqq t \leqq \sigma$ and with the uniform norm $\|\bar{v}\|=$ l.u.b. $_{0 \leqq t \leqq \sigma}|\bar{v}(t)|$. Denoting by $C_{\sigma, M}$ the subspace $\|\bar{v}\| \leqq M$ we shall prove that for some positive $M$ and $\sigma$, and sufficiently small $|\alpha|, \bar{T}$ maps $C_{\sigma, M}$ into itself and is a contraction.

Structure of the proof. The transformation $\bar{w}=\bar{T} \bar{v}(\|\bar{v}\| \leqq M)$ can be written in the form

$$
\bar{w}=\bar{T} \bar{v}=E_{1}(\bar{v})+E_{2}(\bar{v})+E_{3}(\bar{v})
$$

where $E_{1}(\bar{v})$ denotes the sum of the first two terms on the right side of (3.1), $E_{3}(\bar{v})$ denotes the last term, and $E_{2}(\bar{v})$ denotes the rest of the terms. We shall prove that for sufficiently small $\sigma>0$

$$
\left\|E_{1}(\bar{v})\right\| \leqq A_{1}, \quad|| E_{2}(\bar{v})\left\|\leqq A_{2}|\alpha| \sigma^{\frac{1}{2}}, \quad|| E_{3}(\bar{v})\right\| \leqq A_{3}|\alpha| M^{2}
$$

where $A_{i}$ are constants independent of the particular $\bar{v}$ in $C_{\sigma, M}$ and $A_{1}, A_{3}$ are even independent of $M$. Thus, for instance, taking $M=A_{1}+1$ and taking $\sigma$ sufficiently small so that $A_{2} \sigma^{\frac{3}{2}}<\frac{1}{2}$ and $|\alpha|$ sufficiently small so that $A_{3}\left(A_{1}+1\right)^{2}$ $|\alpha|<\frac{1}{2}$, we get $\|\bar{T} \bar{v}\| \leqq M$.

We shall also prove that for any two functions $\bar{v}$ and $\bar{v}_{0}$ in $C_{\sigma, M}$ (and for small $\sigma$ )

$$
\begin{gathered}
\left\|E_{1}(\bar{v})-E_{1}\left(\bar{v}_{0}\right)\right\| \leqq A_{4}|\alpha|\left\|\bar{v}-\bar{v}_{0}\right\|, \\
\left\|E_{2}(\bar{v})-E_{2}\left(\bar{v}_{0}\right)\right\| \leqq A_{5}|\alpha| \sigma^{\frac{3}{2}}|| \bar{v}-\bar{v}_{0} \|, \\
\left\|E_{3}(\bar{v})-E_{3}\left(\bar{v}_{0}\right)\right\| \leqq A_{6}|\alpha|\left\|\bar{v}-\bar{v}_{0}\right\|
\end{gathered}
$$


where $A_{i}$ are constants independent of the particular $\bar{v}$, $\bar{v}_{0}$ in $C_{\sigma, M}$. Thus, for instance, if $\sigma$ satisfies $A_{5} \sigma^{\frac{1}{2}}<\frac{1}{2}$ and $|\alpha|$ is so small that $\left(A_{4}+A_{6}\right)|\alpha|<\frac{1}{2}$, then $\bar{T}$ is a contraction.

The transformation $\bar{w}=\bar{T} \bar{v}$ is essentially a "Volterra type" transformation depending nonlinearly on $\bar{v}$; more precisely, $\bar{w}(t)=(\bar{T} \bar{v})(t)$ depends linearly on $\bar{v}(\tau)$ and nonlinearly on $s(\tau)$ where $0 \leqq \tau \leqq t$, whereas $s(\tau)$ depends on $\bar{v}(\tau)$ by (3.2). The kernels in the integrals in $\bar{T}$ have an integrable singularity.

In deriving bounds on the $E_{i}(\bar{v})$ and $E_{i}(\bar{v})-E_{i}\left(\bar{v}_{0}\right)$ we use the important fact that $s(t)$ is given by an integral involving $\bar{v}(t)$. This point is decisive for the inequalities (3.4), (3.5) and (3.13)-(3.17) which hold for small $\sigma$.

The method presented below would work well for more general boundary relations than (1.9) between $s(t)$ and $u_{x}(s(t), t)=t^{-\frac{1}{2}} \bar{v}(t)$, provided one can establish inequalities of the same character as (3.4), (3.5) and (3.13)-(3.17) and provided the kernels in the integrals of the transformation have singularities of the same type as in $\bar{T}$. As an example we shall consider in Part III the case of one bubble of gas in a liquid of its own substance.

In what follows $B_{i}=B_{i}(\alpha, b, \sigma, M)$ will be used to denote appropriate positive functions, continuous in $\alpha, b, \sigma, M$ provided $b \neq 0$.

3.1. $\bar{T}$ maps $C_{\sigma, m}$ into itself. If we choose $\sigma$ so that

$$
2|\alpha| \sigma+4|\alpha| \sigma^{\frac{1}{2}} M \leqq \frac{1}{2} b^{2},
$$

then on using (3.2) and the inequality $\|\bar{v}\| \leqq M$ we get

$$
\frac{b}{2} \leqq s(t) \leqq \frac{3 b}{2} \text {. }
$$

We shall also need the following inequality:

$$
|s(t)-s(\tau)| \leqq \frac{6|\alpha| M}{b t^{\frac{1}{2}}}(t-\tau), \quad 0<\tau \leqq t \leqq \sigma,
$$

provided

$$
\sigma^{\frac{1}{2}} \leqq M
$$

The proof of (3.5) follows from

$$
\begin{aligned}
&|s(t)-s(\tau)|=\left|\int_{\tau}^{t} \dot{s}(\lambda) d \lambda\right|=\left|\int_{\tau}^{t}\left[\alpha \frac{\bar{v}(\lambda)}{\lambda^{\frac{3}{3}}}-\alpha\right] \frac{d \lambda}{s(\lambda)}\right| \\
& \leqq \frac{2|\alpha| M}{b} \int_{\tau}^{t} \frac{d \lambda}{\lambda^{\frac{1}{3}}}+\frac{2|\alpha|}{b}(t-\tau)
\end{aligned}
$$

and

$$
\int_{\tau}^{t} \frac{d \lambda}{\lambda^{\frac{1}{2}}}=2\left(t^{\frac{1}{2}}-\tau^{\frac{1}{2}}\right) \leqq \frac{2}{t^{\frac{1}{2}}}(t-\tau)
$$

where we made use of (3.4). 
With the aid of (3.4), (3.5) it is now easy to estimate $\|\bar{w}\|$. We have

$$
\|\bar{w}\| \leqq \frac{b}{\pi^{\frac{1}{2}}}+2\|\varphi\|+|\alpha| B_{1} \sigma^{\frac{1}{2}}+12 \frac{|\alpha| M^{2}}{\pi^{\frac{1}{2}} b}
$$

We define

$$
M=2 \frac{b+\|\varphi\| \pi^{\frac{2}{2}}}{\pi^{\frac{1}{2}}}
$$

and assume that $\alpha$ satisfies

$$
\frac{48|\alpha|}{\pi b}\left(b+\|\varphi\| \pi^{\frac{1}{2}}\right)^{2} \leqq \theta b \quad(0<\theta<1)
$$

for some $\theta$. If $\sigma$ satisfies

$$
B_{1} \sigma^{\frac{1}{2}}<\frac{(1-\theta) b}{\pi^{\frac{1}{2}}}
$$

then it follows from (3.7) and (3.8), (3.9) that $\|\bar{w}\| \leqq M$, that is, $\bar{T}$ maps $C_{\sigma, M}$ into itself.

3.2. $\bar{T}$ is a contraction. Let $\bar{w}=\bar{T} \bar{v}, \bar{w}_{0}=\bar{T} \bar{v}_{0}$ and denote

$$
\left\|\bar{v}-\bar{v}_{0}\right\|=\epsilon \text {. }
$$

Let $s_{0}(t)$ be defined by (3.2) with $\bar{v}, s$ replaced by $\bar{v}_{0}, s_{6}$. Since

$$
\|\bar{v}\| \leqq M, \quad\left\|\vec{v}_{0}\right\| \leqq M,
$$

we find as in 3.1 that

$$
\frac{b}{2} \leqq s(t) \leqq \frac{3 b}{2}, \quad \frac{b}{2} \leqq s_{0}(t) \leqq \frac{3 b}{2} .
$$

Using (3.13) and (1.9) we get

$$
t^{\frac{1}{2}}|\dot{s}(t)| \leqq \frac{4|\alpha| M}{b}, \quad t^{\frac{1}{2}}\left|\dot{s}_{0}(t)\right| \leqq \frac{4|\alpha| M}{b} .
$$

We also find, as in 3.1 , that

$$
|s(t)-s(\tau)| \leqq \frac{6|\alpha| M}{b t^{\frac{3}{3}}}(t-\tau), \quad\left|s_{0}(t)-s_{0}(\tau)\right| \leqq \frac{6|\alpha| M}{b t^{\frac{1}{2}}}(t-\tau) .
$$

Next we have

$$
\left|s(t)-s_{0}(t)\right|=\frac{\left|s^{2}(t)-s_{0}^{2}(t)\right|}{s(t)+s_{0}(t)} \leqq \frac{2|\alpha|}{b} \int_{0}^{t} \frac{\left|\bar{v}(\tau)-\bar{v}_{0}(\tau)\right|}{\tau^{\frac{1}{2}}} d \tau .
$$

Hence

$$
\left|s(t)-s_{0}(t)\right| \leqq \frac{4|\alpha|}{b} \epsilon t^{\frac{1}{2}}
$$


We shall also need the inequality

$$
\left|\dot{s}(t)-\dot{s}_{0}(t)\right| \leqq \frac{6|\alpha| \epsilon}{b t^{\frac{1}{2}}} .
$$

To prove (3.17) we first use (3.2) to conclude that

$$
\left|s(t) \dot{s}(t)-s_{0}(t) \dot{s}_{0}(t)\right|=\frac{|\alpha|}{t^{\frac{1}{2}}}\left|\bar{v}(t)-\bar{v}_{0}(t)\right| \leqq \frac{|\alpha| \epsilon}{t^{\frac{1}{2}}} .
$$

Next, using (3.14) and (3.16), we get

$$
\left|s(t) \dot{s}(t)-s_{0}(t) \dot{s}(t)\right|=|\dot{s}(t)|\left|s(t)-s_{0}(t)\right| \leqq \frac{4|\alpha| M}{b t^{\frac{1}{2}}} \frac{4|\alpha|}{b} \epsilon t^{\frac{1}{2}}=16 \frac{\alpha^{2} M \epsilon}{b^{2}} .
$$

Combining (3.18), (3.19) and using (3.13) we conclude that

$$
\left|\dot{s}(t)-\dot{s}_{0}(t)\right| \leqq \frac{2}{b}\left(\frac{|\alpha| \epsilon}{t^{\frac{1}{2}}}+16 \frac{\alpha^{2} M \epsilon}{b^{2}}\right) \leqq \frac{6|\alpha| \epsilon}{b t^{\frac{3}{2}}},
$$

where in the last inequality we made use of (3.3).

We now proceed to estimate $\bar{w}-\bar{w}_{0}$. We write

$$
w-w_{0}=\sum_{i=1}^{5} V_{i}
$$

where

$$
\begin{aligned}
& V_{1}=-2 b t^{\frac{1}{2}}\left[K(s(t), t ; b, 0)-K\left(s_{0}(t), t ; b, 0\right)\right], \\
& V_{2}=-\int_{b}^{\infty} t^{\frac{1}{2}}\left[\frac{s(t)-\xi}{t} K(s(t), t ; \xi, 0)-\frac{s_{0}(t)-\xi}{t} K\left(s_{0}(t), t ; \xi, 0\right)\right] \varphi(\xi) d \xi \\
& V_{3}=2 \alpha \int_{0}^{t} t^{\frac{1}{2}}\left[K(s(t), t ; s(\tau), \tau) \frac{1}{s(\tau)}-K\left(s_{0}(t), t ; s_{0}(\tau), \tau\right) \frac{1}{s_{0}(\tau)}\right] d \tau, \\
& V_{4}=-2 \alpha \int_{0}^{t} t^{\frac{1}{2}}\left[K(s(t), t ; s(\tau), \tau) \frac{\bar{v}(\tau)}{\tau^{\frac{1}{2}} s(\tau)}-K\left(s_{0}(t), t ; s_{0}(\tau), \tau\right) \frac{\bar{v}_{0}(\tau)}{\tau^{\frac{1}{2}} s_{0}(\tau)}\right] d \tau, \\
& V_{5}=\int_{0}^{t} t^{\frac{1}{2}}\left[\frac{s(t)-s(\tau)}{t-\tau} K(s(t), t ; s(\tau), \tau) \frac{\bar{v}(\tau)}{\tau^{\frac{1}{2}}}\right. \\
& \left.\quad-\frac{s_{0}(t)-s_{0}(\tau)}{t-\tau} K\left(s_{0}(t), t ; s_{0}(\tau), \tau\right) \frac{\bar{v}_{0}(\tau)}{\tau^{\frac{1}{2}}}\right] d \tau .
\end{aligned}
$$

Of the five terms to be estimated only $V_{1}$ can be estimated directly by the mean value theorem. The integrand in $V_{2}$ has to be divided into several parts before one can use the mean value theorem. In both of these estimations, the fact that $\sigma$ is small is not enough; $|\alpha|$ has also to be small (see (3.21), (3.27)).

The estimations of $V_{3}$ and $V_{4}$ are somewhat similar to each other. On the one hand, they are more involved since the use of the mean value theorem does not give precise enough results. On the other hand, curiously enough, the final 
results ((3.35) and (3.39)) show that the smallness of $|\alpha|$ need not be used; it is enough to assume that $\sigma$ is small.

The estimation of $V_{5}$, however, is rather delicate and its success is crucial to the validity of the method.

Estimation of $V_{1}$ and $V_{2}$. Using the mean value theorem and (3.16), we get

$$
\left|V_{1}\right| \leqq 2\left(\frac{2}{\pi e}\right)^{\frac{1}{2}}|\alpha| \epsilon \leqq|\alpha| \epsilon .
$$

Next write $V_{2}=V_{2}^{\prime}+V_{2}^{\prime \prime}$ where

$$
\begin{aligned}
V_{2}^{\prime} & =-\int_{b}^{\infty} t^{\frac{1}{2}} \frac{s(t)-s_{0}(t)}{t} K(s(t), t ; \xi, 0) \varphi(\xi) d \xi \\
V_{2}^{\prime \prime} & =-\int_{b}^{\infty} t^{\frac{1}{2}} \frac{s_{0}(t)-\xi}{t}\left[K(s(t), t ; \xi, 0)-K\left(s_{0}(t), t ; \xi, 0\right)\right] \varphi(\xi) d \xi .
\end{aligned}
$$

Using (3.16) one easily gets

$$
\left|V_{2}^{\prime}\right| \leqq \frac{4|\alpha|\|\varphi\|}{b} \epsilon
$$

To estimate $V_{2}^{\prime \prime}$ we use the technique used in estimating $V_{2}^{\prime}$ in Part I, §5. It will be enough to consider only the case $b \leqq s(t) \leqq s_{0}(t)$. Divide the integral of $V_{2}^{\prime \prime}$ into three parts, namely,

$$
-V_{2}^{\prime \prime}=\int_{b}^{\infty}=\int_{b}^{s(t)}+\int_{s(t)}^{s_{0}(t)}+\int_{s_{0}(t)}^{\infty}=S_{1}+S_{2}+S_{3} .
$$

The estimation of $S_{2}$ is immediate. Indeed,

$$
\left|S_{2}\right| \leqq\left|s_{0}(t)-s(t)\right|^{2} \frac{1}{\pi^{\frac{3}{2}} t}\|\varphi\| \leqq 16 \frac{\alpha^{2} M}{b^{2} \pi^{\frac{1}{2}}}\|\varphi\| \epsilon .
$$

Here we made use of the inequalities

$$
\left|s_{0}(t)-\xi\right| \leqq\left|s_{0}(t)-s(t)\right|, \quad|K(x, t ; \xi, 0)| \leqq \frac{1}{2 \pi^{\frac{1}{2}} t^{\frac{1}{2}}}
$$

and of (3.16).

Using the mean value theorem and the inequalities

$$
x \sqrt{2} e^{-x^{2}} \leqq e^{-\frac{1}{2} x^{2}}, \quad|\tilde{s}-\xi| \geqq\left|s_{0}(t)-\xi\right|,
$$

provided $s(t)<\tilde{s}<s_{0}(t)$, we get, after some calculations,

$$
\left|S_{3}\right| \leqq \frac{8 \sqrt{2}|\alpha|}{b}\|\varphi\| \epsilon .
$$

To estimate $S_{1}$ we write in the integrand of $S_{1}$

$$
s_{0}(t)-\xi=\left[s_{0}(t)-s(t)\right]+[s(t)-\xi] .
$$


We then obtain two integrals. The one that corresponds to $[s(t)-\xi]$ can be estimated as $S_{3}$. The second integral is bounded by

$$
\frac{4|\alpha| \epsilon}{b} \int_{b}^{\infty}\left|K(s(t), t ; \xi, 0)+K\left(s_{0}(t), t ; \xi, 0\right)\right||\varphi(\xi)| d \xi \leqq \frac{8|\alpha| \epsilon}{b}\|\varphi\| \text {. }
$$

Combining these last estimates with (3.25), (3.24) and (3.23) we get

$$
\left|V_{2}^{\prime \prime}\right| \leqq 16 \frac{\alpha^{2} M}{b^{2} \pi^{\frac{3}{2}}}\|\varphi\| \epsilon+\left(16 \frac{\sqrt{2}|\alpha|}{b}+8 \frac{|\alpha|}{b}\right)\|\varphi\| \epsilon .
$$

Combining (3.26) with (3.22) we conclude that

$$
\left|V_{2}\right| \leqq 16 \frac{\alpha^{2} M}{b^{2} \pi^{\frac{1}{2}}}\|\varphi\| \epsilon+35 \frac{|\alpha|\|\varphi\|}{b} \epsilon
$$

Estimation of $V_{3}$. Write $V_{3}=V_{3}^{\prime}+V_{3}^{\prime \prime}$ where

$$
\begin{aligned}
V_{3}^{\prime} & =2 \alpha \int_{0}^{t} t^{\frac{1}{2}}\left[K(s(t), t ; s(\tau), \tau)-K\left(s_{0}(t), t ; s_{0}(\tau), \tau\right)\right] \frac{d \tau}{s(\tau)}, \\
V_{3}^{\prime \prime} & =2 \alpha \int_{0}^{t} t^{\frac{1}{2}} K\left(s_{0}(t), t ; s_{0}(\tau), \tau\right)\left[\frac{1}{s(\tau)}-\frac{1}{s_{0}(\tau)}\right] d \tau .
\end{aligned}
$$

To estimate $V_{3}^{\prime}$ consider first the expression

$$
F \equiv 1-\exp \left\{-\frac{\left(s_{0}(t)-s_{0}(\tau)\right)^{2}-(s(t)-s(\tau))^{2}}{4(t-\tau)}\right\}
$$

The expression in the braces is bounded by

$$
\begin{aligned}
\frac{1}{4(t-\tau)}\left[\left|s_{0}(t)-s(t)\right|+\right. & \left.\left|s_{0}(\tau)-s(\tau)\right|\right] \\
\cdot & {\left[\left|s_{0}(t)-s_{0}(\tau)\right|+|s(t)-s(\tau)|\right] \leqq \frac{24 \alpha^{2} M \epsilon}{b^{2}} . }
\end{aligned}
$$

Here we made use of (3.15), (3.16). For later purposes we shall assume that $\alpha$ satisfies

$$
48 \frac{\alpha^{2} M^{2}}{b^{2}} \leqq \frac{1}{2}
$$

Using (3.29) and the inequality $\epsilon \leqq 2 M$ we then conclude that

$$
|F| \leqq e^{\frac{1}{2}} \frac{24 \alpha^{2} M \epsilon}{b^{2}}
$$

If we substitute (3.31) into $V_{3}^{\prime}$, we get

$$
\left|V_{3}^{\prime}\right|=2|\alpha|\left|\int_{0}^{t} t^{\frac{1}{3}} K(s(t), t ; s(\tau), \tau) \frac{F}{s(\tau)} d \tau\right| \leqq B_{2}|\alpha| \sigma \epsilon .
$$


To estimate $V_{3}^{\prime \prime}$ we first note that

$$
\left|\frac{1}{s(\tau)}-\frac{1}{s_{0}(\tau)}\right| \leqq \frac{4}{b^{2}}\left|s(\tau)-s_{0}(\tau)\right| \leqq \frac{16|\alpha| \epsilon}{b^{3}} \tau^{\frac{1}{2}}
$$

Substituting (3.33) into $V_{3}^{\prime \prime}$ we get

$$
\left|V_{3}^{\prime \prime}\right| \leqq B_{3}|\alpha| \sigma \epsilon .
$$

Combining (3.34) with (3.32) we conclude that

$$
\left|V_{3}\right| \leqq\left(B_{2}+B_{3}\right)|\alpha| \sigma \epsilon .
$$

Estimation of $V_{4}$. Write $V_{4}=V_{4}^{\prime}+V_{4}^{\prime \prime}$ where

$$
\begin{aligned}
V_{4}^{\prime} & =-2 \alpha \int_{0}^{t} t^{\frac{1}{2}}\left[K(s(t), t ; s(\tau), \tau)-K\left(s_{0}(t), t ; s_{0}(\tau), \tau\right)\right] \frac{\bar{v}(\tau)}{\tau^{\frac{1}{2}} s(\tau)} d \tau, \\
V_{4}^{\prime \prime} & =-2 \alpha \int_{0}^{t} t^{\frac{1}{2}} K\left(s_{0}(t), t ; s_{0}(\tau), \tau\right)\left[\frac{\bar{v}(\tau)}{s(\tau)}-\frac{\bar{v}_{0}(\tau)}{s_{0}(\tau)}\right] \frac{d \tau}{\tau^{\frac{1}{2}}} .
\end{aligned}
$$

One can estimate $V_{4}^{\prime}$ by the method used in estimating $V_{3}^{\prime}$. Using (3.28), (3.31) one gets

$$
\left|V_{4}^{\prime}\right| \leqq B_{4}|\alpha| \sigma^{\frac{1}{2}} \epsilon
$$

To estimate $V_{4}^{\prime \prime}$ we first note on using (3.13) and (3.33) that

$$
\left|\frac{\bar{v}(\tau)}{s(\tau)}-\frac{\bar{v}_{0}(\tau)}{s_{0}(\tau)}\right| \leqq \frac{2 \epsilon}{b}+16 \frac{|\alpha| M \epsilon}{b^{2}} \tau^{\frac{1}{2}} .
$$

Substituting (3.37) in $V_{4}^{\prime \prime}$ and using (3.3) we obtain

$$
\left|V_{4}^{\prime \prime}\right| \leqq B_{5}|\alpha| \sigma^{\frac{1}{2}} \epsilon \text {. }
$$

Combining (3.36) with (3.38) we get

$$
\left|V_{4}\right| \leqq\left(B_{4}+B_{5}\right)|\alpha| \sigma^{\frac{1}{2}} \epsilon .
$$

Estimation of $V_{5}$. Write

$$
V_{5}=V_{5}^{0}+V_{5}^{\prime}+V_{5}^{\prime \prime}
$$

where

$$
\begin{aligned}
V_{5}^{0} & =\int_{0}^{t} t^{\frac{1}{2}} \frac{s(t)-s(\tau)}{t-\tau} K(s(t), t ; s(\tau), \tau)\left[\bar{v}(\tau)-\bar{v}_{0}(\tau)\right] \frac{d \tau}{\tau^{\frac{1}{2}}} \\
V_{5}^{\prime} & =\int_{0}^{t} t^{\frac{1}{2}}\left[\frac{s(t)-s(\tau)}{t-\tau}-\frac{s_{0}(t)-s_{0}(\tau)}{t-\tau}\right] K(s(t), t ; s(\tau), \tau) \frac{\bar{v}_{0}(\tau)}{\tau^{\frac{3}{2}}} d \tau \\
V_{5}^{\prime \prime} & =\int_{0}^{t} t^{\frac{1}{2}} \frac{s_{0}(t)-s_{0}(\tau)}{t-\tau}\left[K(s(t), t ; s(\tau), \tau)-K\left(s_{0}(t), t ; s_{0}(\tau), \tau\right)\right] \frac{\bar{v}_{0}(\tau)}{\tau^{\frac{1}{2}}} d \tau .
\end{aligned}
$$


Using (3.15) we easily get

$$
\left|V_{5}^{0}\right| \leqq \frac{12}{\pi^{\frac{1}{2}}} \frac{|\alpha| M}{b} \epsilon .
$$

We next divide the integral of $V_{5}^{\prime}$ into two integrals

$$
V_{5}^{\prime}=\int_{0}^{t}=\int_{0}^{\frac{1}{2} t}+\int_{\frac{1}{2} t}^{t}=N_{1}+N_{2} .
$$

Using (3.16) one obtains

$$
\left|N_{1}\right| \leqq \frac{16}{\pi^{\frac{1}{2}}} \frac{|\alpha| M}{b} \epsilon
$$

Using the mean value theorem and (3.17) we get

$$
\left|N_{2}\right| \leqq \frac{6 \sqrt{2}}{\pi^{\frac{1}{2}}} \frac{|\alpha| M}{b} \epsilon .
$$

Combining (3.44), (3.43) with (3.42) we find that

$$
\left|V_{5}^{\prime}\right| \leqq \frac{16+6 \sqrt{2}}{\pi^{\frac{1}{2}}} \frac{|\alpha| M}{b} \epsilon .
$$

It remains to estimate $V_{5}^{\prime \prime}$. Using (3.15) and the inequality (3.31) we find, after some calculation, that

$$
\left|V_{5}^{\prime \prime}\right| \leqq 288\left(\frac{e}{\pi}\right)^{\frac{1}{2}} \frac{|\alpha|^{3} M^{3} \epsilon}{b^{3}}
$$

Combining (3.46), (3.45), (3.41) with (3.40) we conclude that

$$
\left|V_{5}\right| \leqq 26 \frac{|\alpha| M}{b} \epsilon+240 \frac{|\alpha|^{3} M^{3}}{b^{3}} \epsilon .
$$

Combining (3.47), (3.39), (3.35), (3.27), (3.21) with (3.20) we get

$$
\begin{aligned}
\frac{\left\|\bar{w}-\bar{w}_{0}\right\|}{\epsilon} \leqq|\alpha|+10 \frac{\alpha^{2} M}{b^{2}} & \|\varphi\|+35 \frac{|\alpha||| \varphi \|}{b} \\
& +\left(26 \frac{|\alpha| M}{b}+240 \frac{|\alpha|^{3} M^{3}}{b^{3}}\right)+B_{7}|\alpha| \sigma^{\frac{1}{2}}
\end{aligned}
$$

We recall that in proving (3.48) we assumed that $\alpha$ satisfies (3.30).

3.3 Conclusion. It remains to find the limitation on $\alpha$ imposed by (3.9) and (3.30) and by the requirement that $\bar{T}$ be a contraction. We remark here that the previous estimates are not sharp and can be improved. A more precise evaluation of $\alpha$ was sacrificed for the sake of simplicity of calculations. Proceeding with this point of view we shall now prove that if 


$$
|\alpha|<\frac{1}{200}, \quad|\alpha| \frac{\|\varphi\|}{b}<\frac{1}{200}, \quad|\alpha| \frac{\|\varphi\|^{2}}{b^{2}}<\frac{1}{150},
$$

then $\bar{T}$ maps $C_{\sigma, M}$ into itself and is a contraction.

Indeed, using the definition of $M$ in (3.8) we easily find that

$$
\frac{|\alpha| M}{b}<\frac{1}{50}
$$

and, in particular, (3.30) holds. Using (3.50) and (3.49) we also find that

$$
\frac{\left\|\bar{w}-\bar{w}_{0}\right\|}{\epsilon} \leqq \frac{3}{4}+B_{7} \sigma^{\frac{1}{2}} .
$$

Thus if $\sigma$ satisfies (in addition to (3.3), (3.6), (3.10))

$$
B_{7} \sigma^{\frac{1}{2}}<\frac{1}{4}
$$

then $\bar{T}$ is a contraction. The condition (3.9) for some $\theta<1$ is also easily verified on using the first and last inequalities of (3.49).

Having proved that $\bar{T}$ maps $C_{\sigma, M}$ into itself and is a contraction, we conclude that there exists a function $\bar{v}(t)$ in $C_{\sigma, M}$ such that $\bar{v}=\bar{T} \bar{v} ; v(t)=t^{-\frac{1}{2}} \bar{v}(t)$ is then a solution of the integral equation (2.5).

If $v_{0}(t)$ is another solution of the integral equation (2.5) and if $\bar{v}_{0}(t)=t^{\frac{1}{2}} v_{0}(t)$ satisfies

$$
\lim _{t \rightarrow 0}\left|\vec{v}_{0}(t)\right|<M,
$$

then $\bar{v}_{0}(t) \equiv \bar{v}(t)$ for $0 \leqq t<\bar{\sigma}$ where $\bar{\sigma}$ is sufficiently small. Using the definition (3.8) of $M$ and (1.9), it follows that if $v_{0}(t)$ is such that

$$
\lim _{t \rightarrow 0} t^{\frac{1}{2}}\left|\dot{s}_{0}(t)\right|<\frac{2|\alpha|}{1+\alpha} \frac{b+\|\varphi\| \pi^{\frac{1}{2}}}{\pi^{\frac{1}{2}} b},
$$

then $u_{0}(x, t) \equiv u(x, t), s_{0}(t) \equiv s(t)$ for $0 \leqq t \leqq \bar{\sigma}$, where $u_{0}, s_{0}$ is the solution of (1.6)-(1.10) corresponding to $v_{0}$. We have thus completed the discussion of the existence and uniqueness of solutions for small time-intervals.

\section{Existence and Uniqueness for All Times in Case of Condensation}

As already remarked in $\$ 2$ the method used in Part I to prove existence and uniqueness for large intervals of time fails in the present case if $\alpha<0$ (but it works well in case $\alpha>0$; see $\S 6$ ) since we cannot find an appropriate $a$ priori bound on $u_{x}(x, t)$ as in Part I. We shall therefore use a different method based on the inequalities derived in $\S 3$ and which involves only an a priori bound on $u(x, t)$.

We first discuss the problem of uniqueness. Suppose first that $\varphi(b)=b$ and perform integration by parts on the second integral on the right side of (2.5). 
We easily find that the sum of the first two terms on the right side of (2.5) is equal to

$$
2 \int_{b}^{\infty} K(s(t), t ; \xi, 0) \dot{\varphi}(\xi) d \xi .
$$

Thus the right side of (2.5) includes only terms which behave regularly at $t=0$. A comparison of the integral equation (2.5) with the corresponding integral equation for $v(t)$ in Part I shows that the methods and results of Part I, $\$ 5$ remain valid with slight changes. Hence there exists a unique solution for small intervals of time, provided $\alpha>-1$. By a solution, in this context, we mean a pair of functions which satisfy the system of equations (1.6)-(1.10) and all the regularity properties mentioned in $\$ 1$ (thus $\dot{s}(t)$ is continuous at $t=0$ and $u(x, t)$ is continuous at $(b, 0))$.

As in Part I we can also prove that the uniqueness of solutions for any interval of time follows from the uniqueness of solutions for small intervals of times. Thus if $\varphi(b)=b$, then there exists at most one solution (in the sense of $\$ 1$ ), whereas if $\varphi(b) \neq b$ and two solutions coincide for some $t=\sigma>0$, then they coincide in the whole t-interval of their existence.

We proceed to discuss the question of existence of solutions for large intervals of time. We shall need a certain result which we state as a lemma.

Lemma 2. If $\varphi(b)=b$ and $\bar{v}(t)$ satisfies $\bar{v}=\bar{T} \bar{v}$ for $0 \leqq t \leqq \sigma$, then $v(t)=t^{-\frac{1}{2}} \bar{v}(t)$ satisfies (2.5) and is continuous for $0 \leqq t \leqq \sigma$.

Proof. As was proved in $\S 3$, if $v_{0}(t)$ is a solution of (2.5) which satisfies (3.53), then $v(t) \equiv v_{0}(t)$ for all sufficiently small $t$. Now, as was mentioned above, if $\varphi(b)=b$ there exists a solution $v_{0}(t)$ of (2.5) continuous also at $t=0$, and it evidently satisfies (3.53). Hence, for $\bar{\sigma}$ sufficiently small, $v(t) \equiv v_{0}(t)(0<t \leqq \bar{\sigma})$. This proves the continuity of $v(t)$ at $t=0$, and the proof of the lemma is easily completed.

Suppose now that we have constructed a solution for all $0 \leqq t<t_{0}$ and assume that

$$
0<R \equiv \lim _{t \rightarrow t_{0}} s(t)<\infty .
$$

Further, assume (compare with (3.49)) that

$$
\begin{gathered}
\frac{|\alpha|}{R} \limsup _{t \rightarrow t_{o}}|u(x, t)|<\frac{1}{200}, \\
\frac{|\alpha|}{R^{2}}\left[\lim _{t \rightarrow t_{o}} \sup |u(x, t)|\right]^{2}<\frac{1}{150} .
\end{gathered}
$$

From the results of $\S 3$ it follows that there exists a positive number $\delta$ such that for any $z<t_{0}$ we can construct a solution $\bar{v}(t)$ to the integral equation $\bar{v}=\bar{T} \bar{v}$ defined by (3.1), but with $0, b, \varphi(\xi)$ replaced by $\bar{t}, s(\bar{t}), u(\xi, \bar{t})$, and that $\bar{v}(t)$ exists for $t \leqq t \leqq t+\delta$. Since $u(s(t), t)=s(t)$, we can use Lemma 2 to conclude that 
$v(t)=(t-t)^{-\frac{1}{2}} \bar{v}(t)$ is continuous at $t=t$. From the uniqueness results proved above it follows (as in Part I) that the $v(t)$ just constructed coincides with the $v(t)$ whose existence was originally assumed for all $0 \leqq t<t_{0}$ in their common interval of existence. Since $\delta$ is independent of $t$, we have thus proved that the solution $v(t)$ (or $u(x, t), s(t)$ ) can be continued above $t_{0}$ to the interval $0 \leqq t<t_{0}+\delta$, as long as (4.2), (4.3), (4.4) are satisfied.

If $\alpha<0$ then $s(t)$ increases in $t$. Condition (4.2) then means that $s(t)$ remains bounded in bounded times. A quantitative result which includes this statement will be proved in $\S 5$. It thus remains to consider the conditions (4.3), (4.4).

If $\alpha<0$ then, by the maximum principle,

$$
|u(x, t)| \leqq \max (s(t), \| \varphi||) .
$$

Substituting (4.5) into (4.3), (4.4) and noting that $s(t) \geqq b$ we obtain the conditions

$$
|\alpha| \max \left(1, \frac{\|\varphi\|}{b}\right)<\frac{1}{200}, \quad|\alpha| \frac{\|\varphi\|^{2}}{b^{2}}<\frac{1}{150}
$$

which coincide with the conditions (3.49).

However, if $\alpha>0$ we get the conditions

$$
\begin{aligned}
R & >200 \alpha \max (b,\|\varphi\|), \\
R^{2} & >150 \alpha \max \left(b^{2},\|\varphi\|^{2}\right),
\end{aligned}
$$

so that the proof of Theorem 2 in that case is not yet completed. We have, however, completed the proof of existence and uniqueness of solutions in case $\alpha<0$ (condensation). In $\$ 6$ we shall complete the proof in case $\alpha>0$ (evaporation).

Remark. The bounds on $|\alpha|$ seem satisfactory from the point of view of physics. Indeed, it is remarked in [3], [4], [5] that in practice $|\alpha|$ may be expected to be of order of magnitude $2 \cdot 10^{-5}$.

$$
\text { 5. An a Priori Bound on } s(t) \text { For } \alpha<0
$$

In this chapter we shall complete the proof of Theorem 2 in case $\alpha<0$ by proving that if a solution $u(x, t), s(t)$ is defined for $0 \leqq t<t_{0}$, then there exist constants $A, B$ depending only on $\alpha, b, \varphi$ such that for $0 \leqq t<t_{0}$

$$
s(t) \leqq A+B t^{\frac{1}{2}} .
$$

Only in this chapter we make use of the assumption that

$$
C \equiv \int_{b}^{\infty}|\varphi(x)| d x \text { is finite. }
$$

We first remark that for every $t<t_{0}$

$$
\lim _{x \rightarrow \infty} \sup \left|u_{x}(x, t)\right|=\lim _{x m \infty} \sup |\dot{\varphi}(x)| \equiv C^{\prime} .
$$


This follows by differentiating (2.2) with respect to $x$ and then carrying out integration by parts in the first integral on the right side.

If we integrate the heat equation (1.6) over the domain $s(\tau)<\xi<K, 0<\tau<t$ and make use of (1.7), (1.8), (1.9), we get

$$
\begin{aligned}
\int_{0}^{t} u_{x}(K, \tau) d \tau-\frac{1}{2 \alpha} s^{2}(t) & +\frac{1}{2 \alpha} b^{2}-t \\
= & \int_{s(t)}^{K} u(x, t) d x+\int_{0}^{t} s(\tau) \dot{s}(\tau) d \tau-\int_{b}^{K} \varphi(x) d x .
\end{aligned}
$$

We proceed to estimate

$$
I=\int_{s(t)+H}^{K}|u(x, t)| d x \quad\left(H=H_{0} t^{\frac{1}{3}}\right)
$$

where $H_{0}$ is a constant to be determined later. Using the integral representation (2.2) of $u(x, t)$ with $u_{x}$ replaced according to (1.9) and noting that $s(t) \geqq s(\tau)$, $x-s(\tau) \geqq x-s(t) \geqq H$, we get

$$
\begin{aligned}
I \leqq \int_{b}^{\infty}\left[\int_{0}^{\infty} K(x, t ; \xi, 0) d x\right]|\varphi(\xi)| d \xi \\
+2 s(t) \int_{s(t)+H}^{\infty}\left[\int_{0}^{t} \frac{1}{(t-\tau)^{\frac{1}{2}}} K(x, 2 t ; s(t), 2 \tau) d \tau\right] d x \\
\quad+\int_{s(t)+H}^{\infty}\left[\int_{0}^{t} K(x, t ; s(t), \tau) d \tau\right] d x \\
+\frac{1}{|\alpha|} \int_{s(t)+H}^{\infty}\left|\int_{0}^{t} K(x, t ; s(\tau), \tau) \frac{d}{d \tau} s^{2}(\tau) d \tau\right| d x=\sum_{i=1}^{4} M_{i}
\end{aligned}
$$

where $M_{i}$ denotes the $i^{\text {th }}$ term on the right side of (5.6). In what follows we shall denote by $C_{i}$ appropriate positive constants depending only on $b, H_{0}$.

Substituting $\rho=|x-\xi| / t^{\frac{1}{2}}$ in the interior integral of $M_{1}$ and using (5.2) we get

$$
M_{1} \leqq C .
$$

To estimate $M_{2}$ we substitute $\rho=x-s(t)$ and note that $\rho \geqq H$. We obtain

$$
\begin{aligned}
M_{2} \leqq s(t) \int_{H}^{\infty}\left[\int_{0}^{t} \frac{\rho^{2}}{t-\tau} \exp \left\{-\frac{\rho^{2}}{8(t-\tau)}\right\} d \tau\right] \frac{d \rho}{\rho^{2}} & \\
& \leqq C_{2} s(t) \int_{H}^{\infty} t \exp \left\{-\frac{\rho^{2}}{9 t}\right\} \frac{d \rho}{\rho^{2}} .
\end{aligned}
$$

Substituting $\sigma=\rho / t^{\frac{1}{2}}$ and noting that $H / t^{\frac{1}{2}}=H_{0}$ we get, on taking $H_{0} \geqq 1$,

$$
M_{2} \leqq C_{3} s(t) t^{\frac{1}{2}} \int_{H_{0}}^{\infty} \frac{1}{\sigma^{2}} \exp \left\{-\frac{\sigma^{2}}{9}\right\} d \sigma \leqq C_{4} t^{\frac{1}{2}} s(t),
$$

and $C_{4} \rightarrow 0$ as $H_{0} \rightarrow \infty$. 
In a similar way we get

$$
M_{3} \leqq C_{5} t \int_{H_{0}}^{\infty} \frac{1}{\sigma} \exp \left\{-\frac{\sigma^{2}}{9}\right\} d \sigma=C_{6} t
$$

and $C_{6} \rightarrow 0$ as $H_{0} \rightarrow \infty$.

To estimate $M_{4}$ we interchange the order of integration and note that $K>0$, $d s^{2}(\tau) / d \tau \geqq 0$. We get

$$
M_{4} \leqq \frac{1}{|\alpha|} \int_{0}^{t}\left[\int_{s(t)+H}^{\infty} K(x, t ; s(\tau), \tau) d x\right] \frac{d}{d \tau} s^{2}(\tau) d \tau
$$

Substituting $\sigma=(x-s(\tau)) /(t-\tau)^{\frac{1}{2}}$ in the inner integral, and noting that $\sigma \geqq(x-s(t)) / t^{\frac{1}{2}} \geqq H_{0}$, we obtain

$$
\begin{aligned}
& M_{4} \leqq \frac{1}{|\alpha|} C_{9} \int_{0}^{t}\left[\int_{H_{0}}^{\infty} \exp \left\{-\frac{1}{4} \sigma^{2}\right\} d \sigma\right] \frac{d}{d \tau} s^{2}(\tau) d \tau \\
& \leqq \frac{1}{|\alpha|} C_{10} \int_{0}^{t} \frac{d}{d \tau} s^{2}(\tau) d \tau \leqq \frac{1}{|\alpha|} C_{10} s^{2}(t),
\end{aligned}
$$

where $C_{10} \rightarrow 0$ as $H_{0} \rightarrow \infty$.

Combining (5.10), (5.9), (5.8), (5.7) with (5.6), we get

$$
I \leqq C+C_{6} t+C_{4} t^{\frac{1}{2}} s(t)+\frac{1}{|\alpha|} C_{10} s^{2}(t) .
$$

We now note that by the maximum principle $|u(x, t)| \leqq \max (\|\varphi\|, s(t))$. Hence

$$
\int_{s(t)}^{s(t)+H}|u(x, t)| d x \leqq H_{0}\|\varphi\| t^{\frac{1}{2}}+H_{0} t^{\frac{1}{2}} s(t) .
$$

The inequalities (5.11), (5.12) give the desired estimate on the first integral on the right side of (5.4). The second integral on the right side of (5.4) is equal to

$$
\frac{1}{2} s^{2}(t)-\frac{1}{2} b^{2} .
$$

Using these results in (5.4), then taking $K \rightarrow \infty$ and using (5.3), we get the inequality

$$
\begin{aligned}
\mid\left(\frac{1}{2|\alpha|}-\frac{1}{2}\right) s^{2}(t)- & \left(\frac{1}{2|\alpha|}-\frac{1}{2}\right) b^{2}-t \mid \leqq 2 C+C^{\prime} t \\
& \quad+C_{6} t+C_{11} H_{0} t^{\frac{1}{2}} s(t)+H_{0}\|\varphi\| t^{\frac{1}{2}}+\frac{1}{|\alpha|} C_{10} s^{2}(t)
\end{aligned}
$$

where $C_{11}$ is a bounded function of $H_{0}$ and $C_{6}, C_{10}$ tend to zero as $H_{0} \rightarrow \infty$.

Taking $H_{0}$ such that

$$
\frac{1}{|\alpha|} C_{10} \leqq \frac{1}{4|\alpha|}-\frac{1}{4}
$$


we obtain

$$
s^{2}(t) \leqq H_{1}+H_{2} t^{\frac{1}{s}} s(t)+H_{3} t
$$

where $H_{i}$ are constants depending on $\alpha, b, \varphi$. Using (5.15) one easily derives (5.1).

Remark. Later on we shall prove results stronger than (5.1) about the behavior of $s(t)$ for large t.

\section{Existence and Uniqueness for All Times in Case of Evaporation.}

To complete the proof of Theorem 2 it remains to prove the existence of a solution in case $\alpha>0, \varphi(b)=b$ as long as $s(t)$ stays positive. To do this we consider the integral equation (2.5) (rather than the equation $\bar{v}=\bar{T} \bar{v}$ ) and replace the first two terms on the right side of (2.5) by (4.1). The integral equation thus obtained is similar in some respects to the integral equation (4.8) of Part I. As in Part I one can prove also here that a unique solution exists for the present integral equation in an interval of length $\sigma$ depending on $\alpha, b,\|\dot{\varphi}\|$, provided $b \neq 0$. We then can easily conclude that in order to complete the proof of Theorem 2 in case $\alpha>0$ we have to prove, for any $t_{0}>0$, the following theorem.

Theorem $A$. If $R=\lim s(t)>0$ as $t \rightarrow t_{0}$ and if $v(t)$ is defined for all $0 \leqq t<t_{0}$, then $v(t)$ is a function bounded on the set $0 \leqq t<t_{0}$.

Indeed, in this case we can also prove (as in Part I) that $\dot{s}(t)$ and $u_{x}(x, t)$ are bounded functions for $s(t)<x<\infty, 0 \leqq t<t_{0}$. Then an argument used in Part I can be applied to prove that we can continue the solution to any interval of time in which $s(t)$ stays positive.

Proof of Theorem A. For any $0<\delta<t_{0}$ we write for $v(t)$ an integral equation analogous to (2.5) (with the first two terms on the right side of (2.5) replaced by (4.1)) with $0, b$ replaced by $t_{0}-\delta, s\left(t_{0}-\delta\right.$ ) (compare with Part I (6.1)). We then conclude that if $t_{0}-\delta \leqq t<t_{0}$,

$$
\begin{array}{rl}
v(t)=B-2 \alpha \int_{t_{0}-\delta}^{t} & K(s(t), t ; s(\tau), \tau) \frac{v(\tau)}{s(\tau)} d \tau \\
& -2 \int_{t_{0}-\delta}^{t} K_{x}(s(t), t ; s(\tau), \tau) v(\tau) d \tau=B+M_{1}+M_{2}
\end{array}
$$

where $M_{i}(i=1,2)$ denotes the $(i+1)^{\text {th }}$ term on the right side of $(6.1)$ and $B$ depends on $t, t_{0}, \delta$, but satisfies

$$
|B| \leqq B_{1}
$$

where $B_{1}$ depends only on $t_{0}, \delta$.

We now note that since $\alpha>0, \dot{s}(t) \leqq 0$. Hence, by (1.9),

$$
\alpha v(t)=s(t) \dot{s}(t)+\alpha \leqq \alpha .
$$


Dividing by $\alpha$ we conclude that

$$
v(t) \leqq 1 \text { for all } 0<t<t_{0} .
$$

Thus it remains to estimate $v(t)$ from below. Denote

$$
\psi(t)=\underset{t_{0}-\delta<r<t}{\text { g.l.b. }} v(\tau) .
$$

We may assume (without loss of generality) that for any small $\delta, \psi(t)$ is a negative function smaller than -1 . We next take $\delta$ to be sufficiently small so that

$$
\frac{1}{2} R<s(t)<\frac{3}{2} R \text { for } t_{0}-\delta<t<t_{0} .
$$

Using (2.6) we get

$$
|s(t)-s(\tau)| \leqq \frac{2 \alpha}{R}(1+|\psi(t)|)(t-\tau) .
$$

We proceed to estimate the $M_{i}$. Upon using (6.5), (6.6) we obtain

$$
\left|M_{1}\right| \leqq \frac{4 \alpha}{R}|\psi(t)| \int_{t_{0}-\delta}^{t} K(s(t), t ; s(\tau), \tau) d \tau \leqq \frac{4 \alpha}{R}|\psi(t)| \delta^{\frac{1}{2}} .
$$

The decisive step is the estimation of $M_{2}$. Write

$$
M_{2}=\int_{t_{0}-\delta}^{t} \frac{s(t)-s(\tau)}{t-\tau} K(s(t), t ; s(\tau), \tau) v(\tau) d \tau .
$$

Noting that $s(t)$ decreases, (6.4) yields

$$
\frac{s(t)-s(\tau)}{t-\tau} v(\tau) \geqq \frac{s(t)-s(\tau)}{t-\tau} .
$$

Substituting (6.10) into (6.9), we get

$$
M_{2} \geqq \int_{t_{0}-\delta}^{t} \frac{s(t)-s(\tau)}{t-\tau} K(s(t), t ; s(\tau), \tau) d \tau \equiv M^{\prime} .
$$

$M^{\prime}$ is a nonpositive function of $t$ and, by (6.7),

$$
\left|M^{\prime}\right| \leqq \frac{2 \alpha}{R}(1+|\psi(t)|) \int_{t_{0}-\delta}^{t} K(s(t), t ; s(\tau), \tau) d \tau \leqq \frac{2 \alpha}{R}(1+|\psi(t)|) \delta^{\frac{1}{3}} .
$$

Substituting (6.12) into (6.11) we obtain

$$
M_{2} \geqq-\frac{2 \alpha}{R}(1+|\psi(t)|) \delta^{\frac{1}{2}}
$$

Combining (6.13), (6.8), (6.2) with (6.1) we obtain

$$
v(t) \geqq-B_{1}-\frac{2 \alpha}{R}+\frac{6 \alpha}{R} \delta^{\frac{1}{2}} \psi(t) .
$$


Taking the minimum on both sides of (6.14) for $t_{0}-\delta \leqq t \leqq t^{\prime}$ and choosing $\delta$ to be such that the coefficient $\psi(t)$ in (6.14) is smaller than $\frac{1}{2}$, we conclude that

$$
\psi\left(t^{\prime}\right) \geqq-2 B_{1} \quad\left(t_{0}-\delta \leqq t^{\prime}<t_{0}\right),
$$

from which it follows that $v(t)$ is bounded from below. We have thus completed the proof of Theorem A.

We remark that the above method fails in case $\alpha<0$. Indeed, in that case (6.4) holds but the inequality in (6.10) is reversed.

Concluding remark. Theorem 2 can be generalized to second order parabolic equations with smooth coefficients as in Part I. It can also be generalized to systems with (1.8) replaced by a more general equation $u(s(t), t)=h(s(t))$ where $h$ is a given function satisfying appropriate conditions. A similar remark applies to the results of $\$ \S 7,8,9$.

Added in proof. If in $\$ 1$ we perform the transformation $u(x, t)=x(z(x, t)-1)$ (instead of $u=x z$ ), then we obtain the condition $u=0$ on $x=s(t)$ (instead of $u=s$ on $x=s(t)$ ). If we assume that $c_{0}(x) \rightarrow g$ (instead of $c_{0}(x) \rightarrow c_{0} \neq g$ ) sufficiently fast as $x \rightarrow \infty$, then the above calculations can be slightly simplified. Furthermore, a careful estimation then shows that Theorem 2 remains true if we replace (3.49) by the weaker condition

$$
\frac{\alpha\|\varphi\|}{b}<\frac{1}{20} \text { where } \varphi=x(\psi-1)
$$

(compare with Part III, to appear in this journal).

\section{Further a Priori Bounds}

We shall need the following assumptions:

$\left(\Phi_{1}\right) \varphi(x)$ satisfies $(\Phi)$ (see $\S 1$ ) and $\dot{\varphi}(x) \rightarrow 0$ as $x \rightarrow \infty$.

$\left(\Phi_{2}\right) \quad \varphi(x)$ satisfies $\left(\Phi_{1}\right)$ and $\varphi(x) \geqq 0$.

Note that the condition $\varphi(x) \geqq 0$ is equivalent to $c_{0}(x) \geqq c_{0}$ in case $\alpha>0$ and to $c_{0}(x) \leqq c_{0}$ in case $\alpha<0$.

Throughout this section we shall assume that a solution $u(x, t), s(t)$ of the system (1.6)-(1.10) is given and that $|\alpha|<1$. If $\alpha$ satisfies the assumptions imposed in Theorem 2 then the existence and uniqueness of a solution is assured. We shall prove the following statements:

(a) If $0<\alpha<1$ and $\varphi(x)$ satisfies $\left(\Phi_{1}\right)$, then $x=s(t)$ intersects $x=0$ at a finite time $t=t_{0}$.

(b) If $0<\alpha<1$ and $\varphi(x)$ satisfies $\left(\Phi_{2}\right)$, then

$$
t_{0} \leqq \frac{1+\alpha}{2 \alpha} b^{2}+\int_{b}^{\infty} \varphi(x) d x
$$


(c) If $-1<\alpha<0$ and $\varphi(x)$ satisfies $\left(\Phi_{2}\right)$, then

$$
s^{2}(t) \geqq b^{2}+\frac{2|\alpha|}{1-|\alpha|} t-\frac{2|\alpha|}{1-|\alpha|} \int_{b}^{\infty} \varphi(x) d x .
$$

Proof of (a). By (5.3) and the assumption $\left(\Phi_{1}\right)$ it follows that the first integral on the left side of (5.4) tends to zero as $K \rightarrow \infty$. Taking $K \rightarrow \infty$ in (5.4) we then obtain

$$
\left(\frac{1}{2 \alpha}+\frac{1}{2}\right) s^{2}(t)+t=\left(\frac{1}{2 \alpha}+\frac{1}{2}\right) b^{2}+\int_{0}^{\infty} \varphi(x) d x-\int_{s(t)}^{\infty} u(x, t) d x .
$$

It is easily proved that the last integral on the right side of (7.3) is estimated by (5.11), (5.12) also in case $\alpha>0$. Recalling that $s(t) \leqq b$ and replacing $s^{2}(t)$ on the left side of (7.3) by zero, we obtain

$$
t \leqq K_{1}+C_{6} t+K_{2} t^{\frac{1}{2}}
$$

where $K_{1}$ is a constant depending on $\alpha, b, \varphi(x)$ and $K_{2}$ is a constant depending on $b, \varphi(x)$. Since $C_{0} \rightarrow 0$ as $H_{0} \rightarrow \infty$, we may take $H_{0}$ such that

$$
C_{6}<\frac{1}{2} \text {. }
$$

We then have

$$
t \leqq 2 K_{1}+2 K_{2} t^{\frac{1}{2}}
$$

from which it follows that the solution $u(x, t), s(t)$ cannot exist for all $t$. Thus there exists a positive number $t_{0}$ such that $s(t) \rightarrow 0$ as $t \rightarrow t_{0} . t=t_{0}$ satisfies the inequality (7.5).

Remark. A careful examination of the constants which appear in the above estimation of $\int|u(x, t)| d x$, combined with (7.3), shows that

$$
2 \alpha t_{0} \rightarrow b^{2} \text { as } \alpha \rightarrow 0 \text {. }
$$

Proof of (b) By the maximum principle [8; Theorem 2] it follows that $u(x, t)$ $\geqq 0$, hence

$$
\int_{s(t)}^{\infty} u(x, t) d x \geqq 0 .
$$

Substituting (7.7) into (7.3) and taking $t \rightarrow t_{0}, s(t) \rightarrow 0$, we obtain the inequality (7.1).

Proof of (c). Using (7.7) and $\alpha=-|\alpha|$, we obtain from (7.3)

$$
\left(\frac{1}{2|\alpha|}-\frac{1}{2}\right) s^{2}(t) \geqq t+\left(\frac{1}{2|\alpha|}-\frac{1}{2}\right) b^{2}-\int_{0}^{\infty} \varphi(x) d x
$$

which proves (7.2).

We conclude this section by quoting the main results concerning bounds on $s(t)$ and $t_{0}$, derived in $[4,5]$ in the special case $\varphi(x) \equiv 0, b=1$. 
If $0<\alpha<0.38$ then [4]

$$
\begin{gathered}
1-2 \alpha\left[t+2\left(\frac{t}{\pi}\right)^{\frac{1}{2}}\right]<s^{2}(t)<1-\frac{2 \alpha}{1+\alpha} t, \\
\frac{1}{2 \alpha}+\frac{2}{\pi}\left[1-\left(1+\frac{\pi}{2 \alpha}\right)^{\frac{1}{2}}\right]<t_{0}<\frac{1+\alpha}{2 \alpha} .
\end{gathered}
$$

If $-0.05<\alpha<0$ then [5]

$$
\begin{gathered}
A+\frac{B}{t^{\frac{1}{2}}} \leqq s(t) \dot{s}(t) \leqq k\left(A+\frac{B}{t^{\frac{3}{3}}}\right), \\
1+2 A t+4 B t^{\frac{3}{2}} \leqq s^{2}(t) \leqq 1+k\left(2 A t+4 B t^{\frac{1}{3}}\right)
\end{gathered}
$$

where $A, B, k$ are positive constants depending only on $|\alpha|$. As a crude approximation one may take

$$
A=|\alpha|, \quad B=\frac{|\alpha|}{\pi^{\frac{1}{2}}} e^{-|\alpha|}, \quad k=1+4.32|\alpha|^{\frac{1}{2}} .
$$

\section{Continuity and Analyticity of the Solution with RESPECT TO $\alpha$}

The purpose of this chapter is to study the differentiability properties of the solution $u(x, t, \alpha), s(t, \alpha)$ with respect to $\alpha$. The main result is that in every domain $D_{\rho}$ defined by

$$
D_{\rho}: \quad 0<t<\rho, \quad s(t, \alpha)<x<\infty,
$$

the solution is an analytic function of $\alpha$, provided $|\alpha| \rho$ is smaller than an appropriate constant. It should be remarked that in physics $|\alpha|$ is small and, therefore, there is a special interest in studying the behavior of the solution for $\alpha \rightarrow 0$. The case $\alpha=0$ is that of equilibrium, that is, $s(t, 0) \equiv b$. We shall consider in detail the case $b=\varphi(b)$, in which $\dot{s}(t, \alpha)=d s(t, \alpha) / d t$ is continuous at $t=0$ and $u(x, t, \alpha)$ is continuous at $x=b, t=0$. The case $b \neq \varphi(b)$ will be discussed briefly. We shall assume that $\varphi(x)$ satisfies the property $(\Phi)$ of $\S 1$, so that Theorem 2 holds.

8.1. Continuity with respect to $\alpha$. The first theorem is concerned with the continuity properties of the solutions in case $\alpha<0$.

Theorem 3. Let $\alpha_{0}$ be any positive number such that (3.49) holds with $|\alpha|=\alpha_{0}$ and let $\rho$ be any positive number. Then $v(t, \alpha), s(t, \alpha), \dot{s}(t, \alpha), u(x, t, \alpha)$ and $u_{x}(x, t, \alpha)$ are continuous functions in $(x, t, \alpha)$ where $(x, t) \varepsilon \bar{D}_{\rho},-\alpha_{0} \leqq \alpha \leqq 0$.

Remark. An analogous theorem can be stated for $\alpha>0$, provided $\alpha \rho$ is smaller than an appropriate constant depending only on $b, \varphi(x)$. This statement however is contained in Theorems 4,5 .

Proof. The inequality (5.13) shows that if $2|\alpha|<1, \alpha<0$, then

$$
s(t, \alpha) \leqq A_{0} t^{\frac{1}{2}}+B_{0}
$$


where $A_{0}, B_{0}$ may be taken to be constants independent of $\alpha$. Denoting $C_{0}=A_{0} \rho^{\frac{1}{2}}+B_{0}$ we conclude that

$$
s(t, \alpha) \leqq C_{0} \text { provided } 0 \leqq t \leqq \rho .
$$

By the estimates of $\S 3$ it follows that $|\bar{v}(t)| \leqq M$ if $0 \leqq t \leqq \sigma$, where $\bar{v}(t)=$ $t^{\frac{3}{2}} v(t, \alpha), M$ is defined by (3.8), and $\sigma$ satisfies (3.3), (3.6), (3.10) and (3.52). We now wish to apply this result with $t=0$ replaced by $t=\delta$ (and then, accordingly, $b, \varphi(x), \bar{v}(t)=t^{\frac{1}{2}} v(t, \alpha)$ are replaced by $\left.s(\delta), u(x, \delta, \alpha),(t-\delta)^{\frac{1}{2}} v(t, \alpha)\right)$. We then have to take

$$
M=\frac{2}{\pi^{\frac{3}{2}}}\left(s(\delta, \alpha)+\pi^{\frac{1}{2}}\|u(x, \delta, \alpha)\|\right) .
$$

Since by the maximum principle

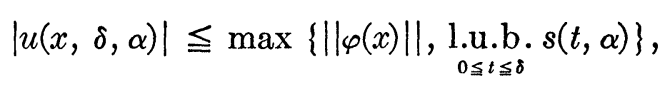

we obtain, on using (8.2),

$$
M \leqq 4 C_{0}+2\|\varphi(x)\| \equiv C_{1} .
$$

We have thus proved that $M$ is bounded by a constant independent of $\alpha$ and $\delta$, provided $0 \leqq \delta<\rho$. By the results of $\S 3$ mentioned before we conclude that for any $\delta, 0 \leqq \delta<\rho$,

$$
(t-\delta)^{\frac{1}{2}}|v(t, \alpha)| \leqq M \leqq C_{1},
$$

provided $0 \leqq t-\delta \leqq \sigma$ and $\sigma$ is restricted by (3.3), (3.6), (3.10), (3.52), where $B_{i}$ are bounded by functions of $\rho, b$ and $M$ only. From the form of these restrictions and the uniform boundedness of $M$ (with respect to $\delta$ and $\alpha$ ) it follows that we may take $\sigma \leqq \beta$ where $\beta$ depends only on $b, \rho,\|\varphi(x)\|$. Taking $t-\delta=\beta$ in (8.3) we conclude that

$$
|v(t, \alpha)| \leqq C_{1} \beta^{-\frac{1}{2}} \equiv C_{2},
$$

provided $\beta \leqq t \leqq \rho$, and $C_{2}$ depends only on $b, \rho,\|\varphi(x)\|$.

We proceed to prove (8.4) for $0 \leqq t \leqq \beta$. It is enough to prove (8.4) for $0 \leqq t \leqq \sigma$ where $\sigma$ is a sufficiently small number depending only on $b$ and $\varphi(x)$.

Denote by $T v$ the right side of (2.5) and replace the first two terms by (4.1). $v(t, \alpha)$ is a fixed point of $T$ in the Banach space $C_{\sigma}$. Consider $T v$ in the space $C_{\sigma, M}(0<\sigma<\rho)$ where $M$ is to be determined later. If $v_{0}(t, \alpha)$ is any function in $C_{\sigma, M}$, that is, if $v_{0}$ is continuous for $0 \leqq t \leqq \sigma$ and $\left\|v_{0}\right\| \leqq M$, then using (2.6) and the method of 3.1 we find that

$$
\left|s_{0}(t, \alpha)-s_{0}(\tau, \alpha)\right| \leqq \frac{2|\alpha|}{b}(M+1)(t-\tau) \quad(0<\tau<t<\sigma)
$$

where $s_{0}(t, \alpha)$ is defined by (2.6) with $v=v_{0}, s=s_{0}$. In deriving (8.5) it is assumed that $\sigma$ is sufficiently small (depending on $b, M$ ). With the aid of (8.5) one finds, using the method of 3.1, that for some positive $M$ independent of $\alpha$, for instance for

$$
M=2\|\dot{\varphi}\|+1,
$$


$T$ maps $C_{\sigma, M}$ into itself, provided $\sigma$ is sufficiently small depending only on $M$, $b, \varphi(x)$. It can also be proved by the method of Part I that $T$ is a contraction. Hence $T$ has a fixed point. From the uniqueness of the solution of the system (1.6)-(1.10) (which is proved by the method of Part I; see also \$4) it follows that the fixed point is $v(t, \alpha)$. Hence

$$
\text { l.u.b. }|v(t, \alpha)| \leqq M \text { for all } \alpha, \quad|\alpha| \leqq \alpha_{0} .
$$

This completes the proof of (8.4) for $0 \leqq t \leqq \rho . C_{2}$ depends on $b, \rho,\|\varphi\|$ and $\|\dot{\varphi}\|$.

Differentiating (2.2) with respect to $\alpha$ and using (2.4), (1.9) and the boundedness of $s(t, \alpha)$ and $v(t, \alpha)$ for $0 \leqq t \leqq \rho$, we get

$$
\left|u_{x}(x, t, \alpha)\right| \leqq C_{3} \text { provided } 0 \leqq t \leqq \rho, \quad s(t)<x<\infty,
$$

where $C_{3}$ is independent of $\alpha$ (it depends only on $b, \varphi(x), \rho$ ).

In the sequel we denote by $A_{i}$ appropriate positive constants depending only on $\rho, b, \varphi(x)$. Let $\alpha, \alpha^{\prime}$ belong to the interval $\left[-\alpha_{0}, 0\right]$ and denote

$$
\epsilon=\epsilon\left(\alpha, \alpha^{\prime}\right)=\left\|v(t, \alpha)-v\left(t, \alpha^{\prime}\right)\right\| .
$$

Using (8.4) and (2.6) we find that

$$
\mid s(t, \alpha)-s\left(t, \alpha^{\prime}\right) \leqq A_{1}\left(|\alpha| \epsilon+\left|\alpha-\alpha^{\prime}\right|\right) t \quad(0 \leqq t \leqq \sigma) .
$$

By the method of Part I we then can prove (compare with (5.11), (5.34) of Part I) that

$$
\operatorname{lius}_{0 \leq t \leq \sigma}\left|v(t, \alpha)-v\left(t, \alpha^{\prime}\right)\right| \leqq A_{2} \sigma^{\frac{1}{2}}\left(|\alpha| \epsilon+\left|\alpha-\alpha^{\prime}\right|\right),
$$

from which it follows that

$$
\left|v(t, \alpha)-v\left(t, \alpha^{\prime}\right)\right| \leqq A_{3}\left|\alpha-\alpha^{\prime}\right| \quad(0 \leqq t \leqq \sigma),
$$

provided $\sigma$ is sufficiently small. $\sigma$ depends only on a lower bound on $b$ and on upper bounds on $b,\|\dot{\varphi}\|, M$.

Having proved that $v(t, \alpha)(0 \leqq t \leqq \sigma)$ is continuous in $\alpha$ uniformly with respect to $t$, we remark that $v(t, \alpha)$ is also continuous in $t$ uniformly with respect to $\alpha$. This follows on using the integral equation (2.5) together with (2.6); details are omitted. Thus $v(t, \alpha)$ is a continuous function in $(t, \alpha)$ for $0 \leqq t \leqq \sigma$, $-\alpha_{0} \leqq \alpha \leqq 0$. The continuity of $s(t, \alpha)$ with respect to $(t, \alpha)$ follows by $(2.6)$, and that of $\dot{s}(t, \alpha)$ follows by (1.9) and the definition

$$
v(t, \alpha)=u_{x}(s(t, \alpha), \alpha) .
$$

By (2.2) and the previous results it follows that also $u(x, t, \alpha)$ is a continuous function of $(x, t, \alpha)$ for $(x, t) \varepsilon \bar{D}_{\sigma},-\alpha_{0} \leqq \alpha \leqq 0$. It remains to consider $u_{x}(x, t, \alpha)$.

Differentiating (2.2) with respect to $x$, making use of (2.4), and integrating by parts the integral

$$
\int_{b}^{\infty} K_{x}(x, t ; \xi, 0) \varphi(\xi) d \xi
$$


we find, recalling that $\varphi(b)=b$, that all we need to prove is that

$$
\Psi(x, t, \alpha)=\int_{0}^{t} K_{x}(x, t ; s(\tau, \alpha), \tau) \psi(\tau, \alpha) d \tau
$$

is uniformly continuous in $(x, t, \alpha)$ for $(x, t) \varepsilon D_{0}$ and $-\alpha_{0} \leqq \alpha \leqq 0$. Here $\psi(\tau, \alpha)$ is a function known to be continuous in $(\tau, \alpha),-\alpha_{0} \leqq \alpha \leqq 0,0 \leqq \tau \leqq \sigma$. Now $\Psi(x, t, \alpha)$ is continuous in $(x, t, \alpha)$, provided $-\alpha_{0} \leqq \alpha \leqq 0$ and $(x, t)$ is restricted to any closed subset of $0 \leqq t \leqq \sigma, s(t, \alpha)<x<\infty$. Furthermore, since $s(t, \alpha)$ satisfies a Lipschitz condition with a coefficient independent of $\alpha$, the proof of Lemma 1 of Part I shows that

$$
\Psi(x, t, \alpha) \rightarrow \int_{0}^{t} K_{x}(s(t, \alpha), t ; s(\tau, \alpha), \tau) \psi(\tau, \alpha) d \tau-\frac{1}{2} \psi(t, \alpha)
$$

as $x \rightarrow s(t, \alpha)+0$, uniformly with respect to $\alpha$ and $t$. Noting that the right side of (8.11) is a continuous function of $(t, \alpha), 0 \leqq t \leqq \sigma,-\alpha_{0} \leqq \alpha \leqq 0$, and recalling that $\Psi(x, t, \alpha)$ is a continuous of $(x, t, \alpha)$ when $(x, t)$ varies in closed subsets of $0 \leqq t \leqq \sigma, s(t)<x<\infty,-\alpha_{0} \leqq \alpha \leqq 0$, we conclude that $\Psi(x, t, \alpha)$ is a uniformly continuous function in $(x, t, \alpha)$ where $(x, t) \varepsilon D_{\sigma},-\alpha_{0} \leqq \alpha \leqq 0$.

Having proved the uniform continuity of the functions $v, s, \dot{s}, u, u_{x}$ with respect to $(x, t, \alpha)$ where $(x, t) \varepsilon D_{\sigma},-\alpha_{0} \leqq \alpha \leqq 0$, we proceed to prove it when $-\alpha_{0} \leqq \alpha \leqq 0$ and $(x, t)$ varies in a domain $D_{\eta}-D_{\sigma}, \eta>\sigma$, using the previous method. The only difference is that now the initial values $u(x, \sigma, \alpha)$ depend on $\alpha$ (whereas $\varphi(x)$ did not depend on $\alpha$ ). However, since by what we have already proved

$$
\underset{8(\sigma) \leqq x<\infty}{\operatorname{l.u.b}}\left|u_{x}(x, \sigma, \alpha)-u_{x}\left(x, \sigma, \alpha^{\prime}\right)\right| \rightarrow 0 \quad \text { as } \quad \alpha \rightarrow \alpha^{\prime},
$$

the previous method can easily be modified to yield the continuity of the functions $v, s, \dot{s}, u, u_{x}$ in a domain $D_{\eta}$. Moreover, by slightly modifying the manner by which $\sigma$ was defined (making use of (8.2), (8.4), (8.6)) it follows that we can take $\eta=2 \sigma$. Proceeding similarly for $D_{3 \sigma}$, etc., the proof of Theorem 3 is completed.

Remark. The method of proof of Theorem 3 can be used to prove, in case $b \neq \varphi(b)$, that $t^{\frac{1}{2}} v(t, \alpha), s(t, \alpha), t^{\frac{1}{2}} \dot{s}(t, \alpha), u(x, t, \alpha)$ and $t^{\frac{1}{2}} u_{x}(x, t, \alpha)$ are continuous functions for $(x, t) \varepsilon \bar{D}_{\rho},-\alpha_{0} \leqq \alpha \leqq 0$.

8.2. Analyticity of $v(t, \alpha), s(t, \alpha) \dot{s}(t, \alpha)$ with respect to $\alpha$. We shall prove the following theorem:

Theorem 4. Assume that $b=\varphi(b)$. There exists a positive constant $\beta$ depending only on $b, \varphi(x)$ such that for every $\sigma>1$ the functions $v(t, \alpha), s(t, \alpha)$ and $\dot{s}(t, \alpha)$ are analytic functions in $\alpha$, for $0 \leqq t \leqq \sigma$, provided $\alpha$ satisfies

$$
|\alpha| \sigma<\beta \text {. }
$$


More precisely, for $0 \leqq t \leqq \sigma$,

$$
v(t, \alpha)=\sum_{n=0}^{\infty} v_{n}(t) \alpha^{n}, \quad s(t, \alpha)=\sum_{n=0}^{\infty} s_{n}(t) \alpha^{n}, \quad \dot{s}(t, \alpha)=\sum_{n=0}^{\infty} \dot{s}_{n}(t) \alpha^{n}
$$

where $\dot{s}_{n}(t)$ is equal to $d s_{n}(t) / d t$, and

$$
\left|v_{n}(t)\right| \leqq K_{0} K^{n}, \quad\left|s_{n}(t)\right| \leqq K_{0} K^{n}, \quad\left|\dot{s}_{n}(t)\right| \leqq K_{0} K^{n}
$$

where $K_{0}$ is an appropriate constant and $K=\sigma / \beta$.

Remark. Later on (in 8.3) we shall also establish the analyticity of $u(x, t, \alpha)$, $u_{*}(x, t, \alpha)$ and derive formulas for the coefficients of the series expansions of $u$ and $s$.

Proof. The proof consists of three parts, namely:

(i) We prove that the nonlinear integral equation (2.5) (with $s$ defined by (2.6)) has a complex solution for $0 \leqq t \leqq \sigma$ provided $\alpha$ is a complex number satisfying (8.13).

(ii) Differentiating formally the equation (2.5) with respect to $\alpha$, we obtain an integral equation for $\partial v / \partial \alpha$, with $\partial s / \partial \alpha$ defined by differentiating (2.6) formally with respect to $\alpha$. We shall prove that this integral equation has a solution for any complex number $\alpha$ satisfying (8.13).

(iii) We shall prove that $\left(v(t, \alpha)-v\left(t, \alpha^{\prime}\right)\right) /\left(\alpha-\alpha^{\prime}\right)$ converges to the solution of the integral equation considered in (ii) which is denoted by $v_{\alpha}(t)$, and that $\left(s(t, \alpha)-s\left(t, \alpha^{\prime}\right)\right) /\left(\alpha-\alpha^{\prime}\right)$ and its $t$-derivative converge to $s_{\alpha}(t)$ and its $t$ derivative (where $s_{\alpha}(t)$ corresponds to $v_{\alpha}(t)$ by differentiating (2.6) with respect $\alpha)$.

If we prove all the assertions of (i), (ii), (iii), then it follows that $v(t, \alpha)$, $s(t, \alpha)$ and $\dot{s}(t, \alpha)$ are complex analytic functions in the complex variable $\alpha$. Therefore (8.14) holds, but with $\dot{s}_{n}(t)$ still being unrelated to $s_{n}(t)$. From the proof of (i), (ii), (iii) it will follow that for all $t, 0 \leqq t \leqq \sigma$, and for all complex numbers $\alpha$ satisfying (8.13),

$$
|v(t, \alpha)| \leqq A_{1}, \quad|s(t, \alpha)| \leqq A_{1}, \quad|\dot{s}(t, \alpha)| \leqq A_{1}
$$

where $A_{1}$ is a constant. Using (8.16) and the Cauchy integral formulas, the proof of (8.15) follows. Finally, using the estimates (8.15) and integrating the series development of $\dot{s}(t, \alpha)$ with respect to $t, 0 \leqq t \leqq \rho$ (for any $\rho<\sigma$ ), we find that $\dot{s}_{n}(t)=d s_{n}(t) / d t$. This completes the proof of Theorem 4 .

In what follows we shall denote by $A$ any positive constant depending only on $b$ and $\varphi(x)$. Occasionally we shall denote $v(t, \alpha), s(t, \alpha)$ by $v(t), s(t)$.

Step (i). The inequalities derived in $\$ 3$ can easily be modified to prove the existence of a solution $v(t, \alpha)$ in every interval $0 \leqq t \leqq \sigma$, provided $\alpha$ is a complex number satisfying (8.13). Thus (3.4) is replaced by

$$
\frac{1}{2} b<|s(t, \alpha)|<\frac{3}{2} b
$$


$C_{\sigma}$ is replaced by the Banach space $X_{\sigma}$ of continuous complex functions $w(t)$ defined in the interval $0 \leqq t \leqq \sigma$ and with the norm $\|w\|=$ l.u.b. $|w(t)|$, and $M$ is taken to be $\sigma^{\frac{3}{2}}$ times an appropriate constant depending only on $b, \varphi(x)$ and $\beta$ (in (8.13)).

Note that since $b=\varphi(b)$ there is no need to introduce in the integral equation (2.5) $\bar{v}=t^{\frac{1}{2}} v$. We also replace the first two terms on the right side of (2.5) by (4.1).

In what follows we shall need the following inequalities:

$$
\begin{gathered}
|v(t, \alpha)| \leqq A \sigma^{\frac{1}{2}}, \\
|s(t, \alpha)-s(\tau, \alpha)| \leqq A \sigma^{\frac{1}{2}}|\alpha|(t-\tau) ;
\end{gathered}
$$

they follow from the above definition of $M$. By the proof of Theorem 3 (see, in particular, (8.8)) which holds also for complex $\alpha$ satisfying (8.13), we obtain, if we evaluate carefully the constant $A_{2}$ in (8.8) and use the equality $M=$ const. $\sigma^{\frac{3}{2}}$, that

$$
\left|v(t, \alpha)-v\left(t, \alpha^{\prime}\right)\right| \leqq A \sigma\left|\alpha-\alpha^{\prime}\right|,
$$

provided $\alpha$ satisfies (8.13). Using (2.6), (8.13) and (8.18), we also get

$$
\begin{gathered}
\left|s(t, \alpha)-s\left(t, \alpha^{\prime}\right)\right| \leqq A \sigma^{\frac{1}{2}}\left|\alpha-\alpha^{\prime}\right| t, \\
\left|\dot{s}(t, \alpha)-\dot{s}\left(t, \alpha^{\prime}\right)\right| \leqq A \sigma^{\frac{1}{2}}\left|\alpha-\alpha^{\prime}\right| .
\end{gathered}
$$

Step (ii). Taking $v(t)=v(t, \alpha), s(t)=s(t, \alpha)$ in (2.5), (2.6), then differentiating formally these equations with respect to $\alpha$ and setting $v_{\alpha}(t)=\partial v(t, \alpha) / \partial \alpha, s_{\alpha}(t)=$ $\partial s(t, \alpha) / \partial \alpha$, we obtain for $v_{\alpha}(t)$ the integral equation

$$
\begin{aligned}
v_{\alpha}(t)=2 s_{\alpha}(t) \int_{b}^{\infty} & K_{x}(s(t), t ; \xi, 0) \dot{\varphi}(\xi) d \xi \\
& +2 \int_{0}^{t} K(s(t), t ; s(\tau), \tau) \frac{1-v(\tau)}{s(\tau)} d \tau \\
& +2 \alpha \int_{0}^{t} K_{x}(s(t), t ; s(\tau), \tau) \frac{1-v(\tau)}{s(\tau)}\left(s_{\alpha}(t)-s_{\alpha}(\tau)\right) d \tau \\
& -2 \alpha \int_{0}^{t} K(s(t), t ; s(\tau), \tau) \frac{v_{\alpha}(\tau)}{s(\tau)} d \tau \\
& -2 \alpha \int_{0}^{t} K(s(t), t ; s(\tau), \tau) \frac{1-v(\tau)}{(s(\tau))^{2}} s_{\alpha}(\tau) d \tau \\
& -2 \int_{0}^{t} K_{x x}(s(t), t ; s(\tau), \tau) v(\tau)\left(s_{\alpha}(t)-s_{\alpha}(\tau)\right) d \tau \\
& -2 \int_{0}^{t} K_{x}(s(t), t ; s(\tau), \tau) v_{\alpha}(\tau) d \tau=\sum_{i=1}^{7} P_{i}(\alpha)
\end{aligned}
$$


where $P_{i}(\alpha)$ denotes the $i^{\text {th }}$ term on the right side of (8.23), and where $s_{\alpha}(t)$ is given by

$$
s_{\alpha}(t)=\frac{1}{s(t)}\left[-t+\int_{0}^{t} v(\tau) d \tau+\alpha \int_{0}^{t} v_{\alpha}(\tau) d \tau\right]
$$

Denote by $X_{\sigma, N}$ the set of functions $w(t)$ in $X_{\sigma}$ (defined above) satisfying $\|w\| \leqq N$. To every $w \varepsilon X_{\sigma, N}$ we define a transformation $S w$ in the following way: Define $r(t)$ by

$$
r(t)=\frac{1}{s(t)}\left[-t+\int_{0}^{t} v(\tau) d \tau+\alpha \int_{0}^{t} w(\tau) d \tau\right]
$$

and replace on the right side of (8.23) $s_{\alpha}$ and $v_{\alpha}$ by $r$ and $w$ respectively. Then the new expression on the right side of (8.23) is denoted by $S w$. If we prove that for any complex $\alpha$ satisfying (8.13) there exists $N$ such that $S$ maps $X_{\sigma, N}$ into itself and is a contraction, then $S$ has a fixed point which we shall denote by $v_{\alpha}(t)$. The corresponding $r(t)$ will be denoted by $s_{\alpha}(t)$.

The proof that $S$ has the above properties is given by the same technique that we used to prove the existence of a solution for $v(t)$ in small time-intervals. It depends on certain $a$ priori inequalities which we proceed to derive.

If $w \varepsilon X_{\sigma, N}$ then $\|w\| \leqq N$. Using (8.17), (8.18) we obtain from (8.25)

$$
\begin{gathered}
|r(t)| \leqq A \sigma^{\frac{1}{2}} t+A|\alpha| N t, \\
\mid r(t)-r(\tau))\left|\leqq A \sigma^{\frac{1}{2}}(t-\tau)+A\right| \alpha \mid N(t-\tau) .
\end{gathered}
$$

Suppose that $w_{0}$ also belongs to $X_{\sigma, N}$ and let $r_{0}(t)$ correspond to it by (8.25). Using (8.25) and (8.17) we obtain

$$
\begin{gathered}
\left|r(t)-r_{0}(t)\right| \leqq A|\alpha| t|| w-w_{0} \|, \\
\left|\dot{r}(t)-\dot{r}_{0}(t)\right| \leqq A|\alpha|\left\|w-w_{0}\right\| .
\end{gathered}
$$

With the aid of (8.26), (8.27) and (8.13), (8.18), (8.19) it follows, after some calculation, that

$$
\|S w\| \leqq A \sigma^{\frac{3}{2}}+A|\alpha| N \sigma .
$$

Hence if we take $N=A \sigma^{\frac{3}{2}}$ and use (8.13) (with $\beta$ sufficiently small), then we conclude that $S$ maps $X_{\sigma, N}$ into itself. Next, using (8.28), (8.29) and (8.13), (8.18), (8.19), it follows, after some calculation, that $S$ is a contraction. The existence of a fixed point $v_{\alpha}(t)$ of $S$ is thus proved.

From the above proof it follows that

$$
\left|v_{\alpha}(t)\right| \leqq A \sigma^{\frac{3}{2}} .
$$

Using (8.24) and (8.13) we get

$$
\left|s_{\alpha}(t)-s_{\alpha}(\tau)\right| \leqq A \sigma^{\frac{1}{2}}(t-\tau) .
$$


Step (iii). Let $\alpha, \alpha^{\prime}$ be distinct complex numbers which satisfy $|\alpha| \sigma<\beta$, $\left|\alpha^{\prime}\right| \sigma<\beta$, and set

$$
\begin{gathered}
v_{\alpha \alpha^{\prime}}(t)=\frac{v(t, \alpha)-v\left(t, \alpha^{\prime}\right)}{\alpha-\alpha^{\prime}}, \quad s_{\alpha \alpha^{\prime}}(t)=\frac{s(t, \alpha)-s\left(t, \alpha^{\prime}\right)}{\alpha-\alpha^{\prime}}, \\
\eta=\left\|v_{\alpha \alpha^{\prime}}-v_{\alpha}\right\| .
\end{gathered}
$$

We have to prove that if $\alpha^{\prime} \rightarrow \alpha$ then $\eta \rightarrow 0,\left\|s_{\alpha \alpha^{\prime}}-s_{\alpha}\right\| \rightarrow 0,\left\|\dot{s}_{\alpha \alpha^{\prime}}-\dot{s}_{\alpha}\right\| \rightarrow 0$. The proof depends on some inequalities which we proceed to derive.

Writing

$$
\begin{aligned}
s_{\alpha \alpha^{\prime}}(t)=\frac{s^{2}(t, \alpha)-s^{2}\left(t, \alpha^{\prime}\right)}{\left(\alpha-\alpha^{\prime}\right)\left[s(t, \alpha)+s\left(t, \alpha^{\prime}\right)\right]} & \\
= & \frac{-2 t+2 \int_{0}^{t} v(\tau) d \tau+2 \alpha^{\prime} \int_{0}^{t} v_{\alpha \alpha^{\prime}}(\tau) d \tau}{s(t, \alpha)+s\left(t, \alpha^{\prime}\right)}
\end{aligned}
$$

and using (8.24), (8.21), (8.31) and (8.13), we get

$$
\left|s_{\alpha \alpha^{\prime}}(t)-s_{\alpha}(t)\right| \leqq A\left|\alpha-\alpha^{\prime}\right| \sigma^{2} t+A|\alpha| \eta t .
$$

Using (8.24), (8.22), (8.31), (8.13) and (8.35), we also get

$$
\left|\dot{s}_{\alpha \alpha^{\prime}}(t)-\dot{s}_{\alpha}(t)\right| \leqq A\left|\alpha-\alpha^{\prime}\right| \sigma^{2}+A|\alpha| \eta \text {. }
$$

With the aid of the inequalities so far obtained (and, in particular, (8.36), (8.37)), we can now proceed to estimate $\eta$. We write (2.5) for $v=v(t, \alpha), v=$ $v\left(t, \alpha^{\prime}\right)$ and subtract one from the other. Upon dividing by $\alpha-\alpha^{\prime}$ we obtain an expression for $v_{\alpha \alpha^{\prime}}(t)$ which is similar to the right side of (8.23); formally it tends to it as $\alpha^{\prime} \rightarrow \alpha$. Subtracting this expression from (8.23) we find that

$$
\left|v_{\alpha \alpha^{\prime}}(t)-v_{\alpha}(t)\right| \leqq \sum_{i=1}^{7}\left|P_{i}\left(\alpha \alpha^{\prime}\right)-P_{i}(\alpha)\right|
$$

where $P_{i}\left(\alpha \alpha^{\prime}\right)$ is the finite-difference analogue of $P_{i}(\alpha)$.

The estimation of each of the terms on the right side of (8.38) is performed by the technique of $\S 3$. We shall derive here only an estimate of the sixth term which is, relatively, the most delicate one. The estimation of the other terms is omitted.

Estimation of $I=\left|P_{6}\left(\alpha \alpha^{\prime}\right)-P_{6}(\alpha)\right|$.

$$
\begin{aligned}
I \leqq 2 & \int_{0}^{t} \mid\left[K_{x}(s(t, \alpha), t ; s(\tau, \alpha), \tau)-K_{x}\left(s\left(t, \alpha^{\prime}\right), t ; s\left(\tau, \alpha^{\prime}\right), \tau\right)\right] \\
& \cdot\left(\alpha-\alpha^{\prime}\right)^{-1}-K_{x x}(s(t, \alpha), t ; s(\tau, \alpha), \tau)\left[s_{\alpha}(t)-s_{\alpha}(\tau)\right]|| v(\tau) \mid d \tau .
\end{aligned}
$$

By the mean value theorem for real functions we find that if $f(z)$ is a complex analytic function, then

$$
f\left(z_{1}\right)-f\left(z_{2}\right)=\left(z_{1}-z_{2}\right) f^{\prime}\left(\tilde{z}_{1}\right)+R\left(\tilde{z}_{1}, \tilde{z}_{2}\right) \quad\left(f^{\prime}(z)=\frac{d f(z)}{d z}\right)
$$


where $\tilde{z}_{1}, \tilde{z}_{2}$ lie in the interval $\left(z_{1}, z_{2}\right)$ and

$$
\left|R\left(\tilde{z}_{1}, \tilde{z}_{2}\right)\right| \leqq\left|z_{1}-z_{2}\right|\left|f^{\prime}\left(\tilde{z}_{1}\right)-f^{\prime}\left(\tilde{z}_{2}\right)\right| \text {. }
$$

Using this remark with $f(z)=K_{z}(z, t ; 0, \tau)$ and using (8.16), (8.32), we obtain from (8.39)

$$
\begin{aligned}
& I \leqq A \int_{0}^{t}\left|K_{x x}\right|\left|\left[s_{\alpha \alpha^{\prime}}(t)-s_{\alpha \alpha^{\prime}}(\tau)\right]-\left[s_{\alpha}(t)-s_{\alpha}(\tau)\right]\right| d \tau \\
& \quad+A \sigma^{\frac{3}{2}} \int_{0}^{t}(t-\tau) \mid K_{x x}(s(t, \alpha), t ; s(\tau, \alpha), \tau) \\
& +A \int_{0}^{t}\left|K_{x x}\left(s_{1}(t), t ; s_{1}(\tau), \tau\right) \quad-K_{x x}\left(s_{1}(t), t ; s_{1}(\tau), \tau\right)\right| d \tau \\
& \quad-K_{x x}\left(s_{2}(t), t ; s_{2}(\tau), \tau\right)|| s_{\alpha \alpha^{\prime}}(t)-s_{\alpha \alpha^{\prime}}(\tau) \mid d \tau \\
& \quad=M_{1}+M_{2}+M_{3}
\end{aligned}
$$

where $M_{i}$ denotes the $i^{\text {th }}$ term on the right side of (8.42) and $s_{i}(t)-s_{i}(\tau)$ lies in the interval $\left(s(t, \alpha)-s(\tau, \alpha), s\left(t, \alpha^{\prime}\right)-s\left(\tau, \alpha^{\prime}\right)\right)$. Using (8.21), we get

$$
\left|s_{i}(t)-s(t, \alpha)\right| \leqq A\left|\alpha-\alpha^{\prime}\right| \sigma^{\frac{3}{2}} t .
$$

Using (8.37) we easily get

(8.44) $M_{1} \leqq A \int_{0}^{t}\left|K_{x x}\right||| \dot{s}_{\alpha \alpha^{\prime}}-\dot{s}_{\alpha} \|(t-\tau) d \tau \leqq A\left|\alpha-\alpha^{\prime}\right| \sigma^{5 / 2}+A|\alpha| \sigma^{1 / 2} \eta$.

Noting that

$$
\left|K_{x x x}(x, t ; \xi, \tau)\right|=\left|K_{t x}(x, t ; \xi, \tau)\right| \leqq A \frac{|x-\xi|}{(t-\tau)^{5 / 2}}
$$

and using the inequality (for $f(z)$ analytic)

$$
\left|f\left(z_{1}\right)-f\left(z_{2}\right)\right| \leqq\left|z_{1}-z_{2}\right| \max _{z \varepsilon\left(z_{1}, z_{2}\right)}\left|f^{\prime}(z)\right|
$$

with $f(z)=K_{z z}(z, t ; 0, \tau)$, we obtain

$$
M_{2} \leqq A \sigma^{\frac{1}{2}} \int_{0}^{t} \frac{1}{(t-\tau)^{\frac{1}{2}}}\left|s_{3}(t)-s_{3}(\tau)\right|\left[\left|s_{1}(t)-s(t, \alpha)\right|+\left|s_{1}(\tau)-s(\tau, \alpha)\right|\right] d \tau .
$$

Here $s_{3}(t)-s_{3}(\tau)$ lies in the interval $\left(s(t, \alpha)-s(\tau, \alpha), s\left(t, \alpha^{\prime}\right)-s\left(\tau, \alpha^{\prime}\right)\right)$, and hence (8.19) holds with $s$ replaced by $s_{3}$ and $|\alpha|$ replaced by $\bar{\alpha}=\max \left\{|\alpha|,\left|\alpha^{\prime}\right|\right\}$. Using (8.19), (8.43) and the last remark, we get

$$
M_{2} \leqq A|\bar{\alpha}| \sigma^{3}\left|\alpha-\alpha^{\prime}\right| .
$$

To estimate $M_{3}$ we note, by (8.32), $(8,37)$, that

$$
\begin{aligned}
\left|s_{\alpha \alpha^{\prime}}(t)-s_{\alpha \alpha^{\prime}}(\tau)\right| \leqq\left|s_{\alpha}(t)-s_{\alpha}(\tau)\right|+(t-\tau)|| \dot{s}_{\alpha}-\dot{s}_{\alpha \alpha^{\prime}} \| \\
\leqq A \sigma^{\frac{1}{2}}(t-\tau)+A\left|\alpha-\alpha^{\prime}\right| \sigma^{2}(t-\tau)+A|\alpha| \eta(t-\tau) .
\end{aligned}
$$


Substituting (8.46) into $M_{3}$ and comparing the integral thus obtained with the integral of $M_{2}$, we easily find that

$$
M_{3} \leqq A|\alpha| \sigma^{3}\left|\alpha-\alpha^{\prime}\right|+A|\alpha| \sigma^{9 / 2}\left|\alpha-\alpha^{\prime}\right|^{2}+A|\alpha|^{2} \sigma^{5 / 2} \eta\left|\alpha-\alpha^{\prime}\right| .
$$

Combining (8.47), (8.45), (8.44) with (8.42) and using (8.13), we obtain

$$
I \leqq A \sigma^{5 / 2}\left|\alpha-\alpha^{\prime}\right|+A|\alpha|^{1 / 2} \eta .
$$

Conclusion. It can be proved that the other terms on the right side of (8.38) are also bounded by the right side of (8.48) plus $A|\bar{\alpha}| \sigma \eta$. Hence, by (8.38) and the definition (8.34),

$$
\eta \leqq A \sigma^{5 / 2}\left|\alpha-\alpha^{\prime}\right|+A|\alpha|^{1 / 2} \eta+A|\bar{\alpha}| \sigma \eta .
$$

We conclude that $\eta<A \sigma^{5 / 2}\left|\alpha-\alpha^{\prime}\right|$. Hence $\eta \rightarrow 0$ as $\alpha^{\prime} \rightarrow \alpha$. By (8.36) and (8.37) it follows that also $\left\|s_{\alpha}-s_{\alpha \alpha}\right\| \rightarrow 0,\left\|\dot{s}_{\alpha}-\dot{s}_{\alpha \alpha}\right\| \rightarrow 0$ as $\alpha^{\prime} \rightarrow \alpha$. The proof of Theorem 4 is thereby completed.

Remark. Since for $\alpha$ real the functions $v(t, \alpha)$ and $s(t, \alpha)$ are real functions, we conclude that in (8.14) the coefficients $v_{n}(t), s_{n}(t)$ are real functions.

8.3. Analyticity of the solution with respect to $\alpha$. Explicit formulas. Since the boundary $s(t, \alpha)$ varies with $\alpha$, it would be difficult to study directly the analyticity of $u(x, t, \alpha)$ near the free boundary. So we first make the transformation

$$
y=x-s(t, \alpha)
$$

which maps the free boundary $x=s(t, \alpha)$ onto the line $y=0$. Upon setting

$$
u(x, t, \alpha)=U(y, t, \alpha), \quad \varphi(y+b)=\Phi(y)
$$

and denoting by $E_{\sigma}$ the set $0<t<\sigma, 0<y<\infty$, the system (1.6)-(1.10) in $D_{\sigma}$ takes the form

$$
\begin{gathered}
U_{y y}-U_{t}+\dot{s}(t, \alpha) U_{y}=0 \text { for }(y, t) \varepsilon E_{\sigma}, \\
U(y, 0, \alpha)=\Phi(y) \text { for } 0 \leqq y<\infty \\
U(0, t, \alpha)=s(t, \alpha) \text { for } 0 \leqq t \leqq \sigma \\
\alpha U_{y}(0, t, \alpha)=s(t, \alpha) \dot{s}(t, \alpha)+\alpha \text { for } 0<t \leqq \sigma \\
U(y, t, \alpha) \text { and } U_{y}(y, t, \alpha) \text { remain bounded as } y \rightarrow \infty \text { uniformly } \\
\text { with respect to } t, 0 \leqq t \leqq \sigma .
\end{gathered}
$$

We shall now prove the following theorem.

Theorem 5. Assume that $\varphi(x)$ satisfies the assumption ( $\Phi)$ of $\$ 1$, that $\left|\partial^{2} \varphi(x) / \partial x^{2}\right| \leqq$ const., and that $\varphi(b)=b$. For every $\sigma>1$ we have, in $E_{\sigma}$,

$$
U(y, t, \alpha)=\sum_{n=0}^{\infty} U_{n}(y, t) \alpha^{n}, \quad\left|U_{n}(y, t)\right| \leqq H_{0} H^{n}
$$




$$
s(t, \alpha)=\sum_{n=0}^{\infty} s_{n}(t) \alpha^{n}, \quad \dot{s}_{n}(t, \alpha)=\sum_{n=0}^{\infty} \dot{s}_{n}(t) \alpha^{n}
$$

where $s_{n}, \dot{s}_{n}$ satisfy (8.15) and where $H$ can be taken as const. $\sigma^{-1}$ (for some const. $>0)$. The functions $U_{n}, s_{n}$ satisfy the equations

$$
\begin{gathered}
\frac{\partial^{2} U_{n}}{\partial y^{2}}-\frac{\partial U_{n}}{\partial t}+\sum_{i=1}^{n} \dot{s}_{i}(t) \frac{\partial}{\partial y} U_{n-i}(y, t)=0 \quad(n=0,1,2, \cdots), \\
U_{n}(y, 0)=0(n=1,2, \cdots) ; \quad U_{0}(y, 0)=\Phi(y), \\
U_{n}(0, t)=s_{n}(t)(n=0,1,2, \cdots) ; \quad s_{0}(t) \equiv b \\
\frac{\partial}{\partial y} U_{0}(0, t)=s_{0}(t) \dot{s}_{1}(t)+1, \\
\frac{\partial}{\partial y} U_{n}(0, t)=\sum_{i=0}^{n} s_{i}(t) \dot{s}_{n+1-i}(t) \quad(n=1,2, \cdots) .
\end{gathered}
$$

Furthermore,

$$
\begin{aligned}
& U_{n}(y, t) \text { and }(\partial / \partial y) U_{n}(y, t)(n=0,1, \cdots) \text { remain bounded as } y \rightarrow \infty, \\
& \text { uniformly with respect to } t, 0<t<\sigma .
\end{aligned}
$$

Proof. Considering $s_{m}(t)(m=0,1, \cdots)$ to be known functions, the system (8.58)-(8.60) together with (8.62) can be used to define $U_{n}$ in terms of the $U_{m}$ $(m=0,1, \cdots, n-1)$. In order to prove the existence of $U_{n}$ and deduce some of its properties needed later, we shall need the following lemma.

Lemma 3. Let $f(y, t)$ satisfy a Hölder condition (exponent $\lambda$ ) with respect to $y$, uniformly in $E_{\sigma}$, let $f(y, t), g(t), \dot{g}(t), h(y), \dot{h}(y) \dddot{h}(y)$ be bounded continuous functions for $0 \leqq t \leqq \sigma, 0 \leqq y<\infty$, and let $h(0)=g(0)$. Then there exists a unique solution of the system

$$
\begin{gathered}
w_{y y}-w_{t}=f(y, t) \text { for }(y, t) \varepsilon E_{\sigma}, \\
w(0, t)=g(t) \text { for } 0 \leqq t \leqq \sigma, \\
w(y, 0)=h(y) \text { for } 0 \leqq y<\infty, \\
w(y, t) \text { and } w_{y}(y, t) \text { are bounded functions in } E_{\sigma} .
\end{gathered}
$$

The solution $w$ satisfies the inequality $\left(\right.$ for $\left.(y, t) \varepsilon E_{s}\right)$

$$
\begin{aligned}
|w|_{0}+\left|w_{y}\right|_{0} & +\left|w_{y y}\right|_{0}+\left|w_{t}\right|_{0}+H_{y}\left(w_{y}\right) \\
& \leqq M_{1}\left[|f|_{0}+H_{y}(f)+|g|_{0}+|\dot{g}|_{0}+|h|_{0}+|\dot{h}|_{0}+|\ddot{h}|_{0}\right]
\end{aligned}
$$

where $M_{1}$ is a constant depending only on $\sigma$, and

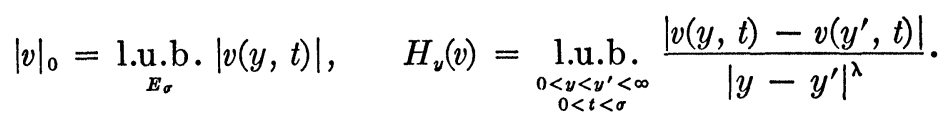


Proof of the lemma. Denote by $G(y, t ; \eta, \tau)=K(y, t ; \eta, \tau)-K(-y, t ; \eta, \tau)$ the Green function of the heat equation in the half space $0<y<\infty$. We shall prove that $w(y, t)$ is given by

$$
\begin{aligned}
w(y, t)=\int_{0}^{t} g(\tau) \frac{\partial}{\partial \eta} G(y, t ; 0, \tau) d \tau & +\int_{0}^{\infty} h(\eta) G(y, t ; \eta, 0) d \eta \\
& +\int_{0}^{t} \int_{0}^{\infty} G(y, t ; \eta, \tau) f(\eta, \tau) d \eta d \tau .
\end{aligned}
$$

Indeed, by [9; p. 52] it follows that the last integral on the right side of (8.68), denoted by $z(y, t)$, satisfies

$$
z_{y y}-z_{t}=f(y, t) .
$$

One can also easily verify that the right side of (8.68) satisfies (8.64)-(8.66). It thus follows that the right side of (8.68) is indeed a solution of (8.63)-(8.66).

To prove uniqueness we first note that every solution must have a continuous $y$-derivative up to the boundary (the proof is similar to that of Part I, §7). Using Green's formula for any possible solution and using (8.63)-(8.66), we conclude that the solution is equal to the right side of (8.68). Hence it coincides with $w$.

Differentiating (8.68) with respect to $y$ and integrating by parts, we obtain

$$
\begin{aligned}
w_{\nu}(y, t)=-\int_{0}^{t} \dot{g}(\tau) N(y, t ; 0, \tau) d & +\int_{0}^{\infty} \dot{h}(\eta) N(y, t ; \eta, 0) d \eta \\
& +\int_{0}^{t} \int_{0}^{\infty} G_{\nu}(y, t ; \eta, \tau) f(\eta, \tau) d \eta d \tau
\end{aligned}
$$

where $N(y, t ; \eta, \tau)=K(y, t ; \eta, \tau)+K(-y, t ; \eta, \tau)$ is the Neumann function of the heat equation in the half plane $0<y<\infty$.

The inequality (8.67) now follows by using (8.68), (8.69) and some known properties concerning fundamental solutions (see, for instance, $[9,10]$ and Lemma 1 of Part I).

Completion of the proof. We shall prove existence of the $U_{n}$ and derive some inequalities on them by induction on $n$. Assume that the $U_{m}(y, t)(m=0,1, \cdots$, $n-1$ ) exist and satisfy (8.58)-(8.60), (8.62), and assume also that

$$
I_{m} \equiv\left|U_{m}\right|_{0}+\left|\frac{\partial}{\partial y} U_{m}\right|_{0}+\left|\frac{\partial^{2}}{\partial y^{2}} U_{m}\right|_{0}+\left|\frac{\partial}{\partial t} U_{m}\right|+H_{\nu}\left(\frac{\partial}{\partial y} U_{m}\right) \leqq H_{0} H^{m} .
$$

We shall prove that $U_{n}$ exists as a solution of (8.58)-(8.60), (8.62), and that (8.70) holds for $m=n$.

Now, the existence of $U_{n}$ follows by Lemma 3. Using (8.67), the inductive assumptions and (8.15), we also have

$$
I_{n} \leqq M_{1} \sum_{i=1}^{n} K_{0} K^{i}\left[\left|\frac{\partial}{\partial y} U_{n-i}\right|_{0}+H_{\nu}\left(\frac{\partial}{\partial y} U_{n-i}\right)\right]+2 M_{1} K_{0} K^{n} .
$$


If we take $H \geqq 2 K$ and use (8.70), we get

$$
I_{n} \leqq M_{1} K_{0} H_{0} H^{n-1} \sum_{i=1}^{n-1} \frac{1}{2^{i}}+\left(H_{0}+2 M_{1}\right) K_{0} K^{n} \leqq M_{2} H_{0} H^{n-1}
$$

where $M_{2}$ is a constant independent of $n$. Taking $H \geqq M_{2}$ the proof of (8.70) for $m=n$ is completed. Note that $H$ can be taken to be const. $K$; hence, by Theorem $4, H=$ const. $\sigma^{-1}$.

The existence of $U_{0}$ satisfying (8.58)-(8.60), (8.62) for $n=0$ and (8.70) for $m=0$ can also be proved very easily using Lemma 3 and taking $H_{0}$ sufficeintly large and $H$ to satisfy $H \geqq 1$. This fixes $H_{0} . H$ is then determined by the (previously proved) passage from $n-1$ to $n$. The proof by induction is thus completed.

Having proved the existence of $U_{n}(y, t)(n=0,1,2, \cdots)$ satisfying (8.58)(8.60), (8.62) and (8.70), we conclude that the function

$$
V(y, t, \alpha)=\sum_{n=0}^{\infty} U_{n}(y, t) \alpha^{n}
$$

can be differentiated in $E_{\sigma}$ term by term twice with respect to $y$ and once with respect to $t$, and $V_{v}(y, t, \alpha)$ is continuous for $(y, t) \varepsilon \bar{E}_{\sigma}$. Here $\alpha$ is taken to satisfy $|\alpha| H<1$. Using (8.58)-(8.60), (8.62) we easily find that $V$ satisfies the equations (8.51)-(8.53) and the condition at infinity (8.55). Thus $U-V$ vanishes on $t=0, y=0$ and satisfies (8.51), (8.55) (with $U$ replaced by $U-V$ ). Denoting $w=U-V$, we proceed to prove that $w \equiv 0$ in $D_{\sigma}$.

By the proof of Lemma 3 we conclude that $w$ is represented by (8.68) with $g(\tau) \equiv 0, h(\eta) \equiv 0$ and $f(\eta, \tau)=-\dot{s}(\tau, \alpha) w_{\eta}(\eta, \tau)$. Differentiating this representation with respect to $y$ we obtain

$$
w_{y}(y, t)=-\int_{0}^{t} \int_{0}^{\infty} \dot{s}(\tau, \alpha) G_{y}(y, t ; \eta, \tau) w_{\eta}(\eta, \tau) d \eta d \tau
$$

This is a Volterra type integral equation, and by iteration we get $w_{y}(y, t) \equiv 0$. Hence $w(y, t)$ is a function of $t$ only. Since $w(0, t) \equiv 0$ we obtain $w(y, t) \equiv 0$.

Having proved that $V(y, t, \alpha)$ coincides with the solution $U(y, t, \alpha)$, we insert (8.71) into (8.54) and thus derive the equations (8.61). The proof of the theorem is thereby completed.

Note that the system (8.58)-(8.62) can be used to construct the coefficients $U_{m}, s_{m}$ step by step. Indeed, if $U_{m}, s_{m}$ have already been constructed for $m=0$, $1, \cdots, n-1$, then $s_{n}$ is defined from (8.61) and $U_{n}$ is defined as a solution of (8.58)-(8.60), (8.62).

Remark. If $\varphi(b) \neq b$ then $t^{\frac{1}{2}} v(t, \alpha), s(t, \alpha), t^{\frac{1}{2}} s(t, \alpha)$ are analytic functions in $\alpha$ for $0 \leqq t \leqq \sigma$; the proof is similar to that of Theorem 4 . Theorem 5 also holds, but the inequalities (8.70) have to be slightly changed.

\section{Asymptotic Behavior of Solutions for $\alpha<0$ as $t \rightarrow \infty$}

In this chapter we shall study the behavior of $s(t, \alpha), \dot{s}(t, \alpha)$ and $u(x, t, \alpha)$ as $t \rightarrow \infty$ in the case of condensation $(\alpha<0)$, provided $|\alpha|$ is sufficiently small. 
9.1. Behavior of $s(t, \alpha)$. Theorem 6. Let $\varphi(x)$ satisfy $\left(\Phi_{1}\right)$ (see §7). Then for every $\eta>0$ there exists $\alpha^{*}=\alpha^{*}(\eta)>0$ ((3.49) is satisfied for $|\alpha|=\alpha^{*}$ and $\alpha^{*}$ depends on $\eta, b, \varphi(x))$ such that if $\alpha<0,|\alpha| \leqq \alpha^{*}$, then the function $s(t, \alpha)$ of the solution $u, s$ of (1.6)-(1.10) satisfies

$$
\limsup _{t \rightarrow \infty}\left|\frac{s^{2}(t, \alpha)}{2|\alpha| t}-1\right| \leqq \eta \text {. }
$$

Proof. Since $\varphi(x)$ satisfies $\left(\Phi_{1}\right), C^{\prime}$ in (5.13) $\left(C^{\prime}\right.$ is defined by (5.3)) is zero. Using the inequalities, obvious for all $\epsilon>0$,

$$
\begin{gathered}
C_{11} H_{0} t^{\frac{1}{s}} s(t) \leqq \epsilon t+\frac{1}{4 \epsilon}\left(C_{11} H_{0}\right)^{2} s^{2}(t), \\
H_{0}\|\varphi\| t^{\frac{1}{2}} \leqq \epsilon t+\frac{1}{4 \epsilon}\left(H_{0}\|\varphi\|\right)^{2},
\end{gathered}
$$

(5.13) takes the form

$$
\begin{aligned}
\mid\left[\frac{1+2 \theta_{0} C_{10}}{2|\alpha|}+\frac{\theta_{1}}{4 \epsilon}\left(C_{11} H_{0}\right)^{2}-\frac{1}{2}\right] s^{2}(t)-\left(1+2 \theta_{2} \epsilon+\theta_{3} C_{6}\right) t \\
-\frac{b^{2}}{2|\alpha|} \mid \leqq 2 C+\frac{b^{2}}{2}+\frac{1}{4 \epsilon} H_{0}^{2}\|\varphi\|^{2}
\end{aligned}
$$

where $\left|\theta_{i}\right| \leqq 1, C_{11}$ is a bounded function of $H_{0}\left(1 \leqq H_{0}<\infty\right)$ and $C_{10}, C_{0}$ tend to zero as $H_{0} \rightarrow \infty$.

Let $\delta<1$ be an arbitrary positive number. Choose $H_{0}$ (which was an arbitrary number $\geqq 1$; see $\$ 5$ ) such that

$$
2 C_{10} \leqq \frac{1}{3} \delta, \quad C_{6} \leqq \frac{1}{3} \delta .
$$

Then take $\epsilon=\frac{1}{3} \delta$ and let $\alpha^{*}\left(|\alpha| \leqq \alpha^{*}\right)$ satisfy

$$
\frac{3}{4 \epsilon}\left(C_{11} H_{0}\right)^{2} \leqq \frac{\delta}{6 \alpha^{*}}, \quad \frac{1}{2} \leqq \frac{\delta}{6 \alpha^{*}} .
$$

Combining these inequalities we conclude that the coefficient of $s^{2}(t)$ in (9.2) lies between the two numbers $(1 \pm \delta) / 2|\alpha|$ and that the coefficient of $t$ in (9.2) lies between the two numbers $1 \pm \delta$. Dividing (9.4) by $t$ we easily get

$$
\begin{aligned}
& \frac{s^{2}(t)}{2|\alpha| t}-1 \leqq \frac{2 \delta}{1-\delta}+\frac{b^{2}}{(1-\delta)|\alpha| t} \\
& +\frac{1}{(1-\delta) t}\left(2 C+\frac{b^{2}}{2}+\frac{3}{4 \delta} H_{0}^{2}\|\varphi\|^{2}\right), \\
& \frac{s^{2}(t)}{2|\alpha| t}-1 \geqq-\frac{2 \delta}{1-\delta}+\frac{b^{2}}{(1+\delta)|\alpha| t} \\
& -\frac{1}{(1-\delta) t}\left(2 C+\frac{b^{2}}{2}+\frac{3}{4 \delta} H_{0}^{2}\|\varphi\|^{2}\right) .
\end{aligned}
$$


Since $\delta$ is an arbitrary positive number, and since $\alpha^{*}$ depends only on $\delta, b, \varphi(x)$, the proof of (9.1) is completed.

9.2. Behavior of $\dot{s}(t, \alpha)$. Theorem 7. Let $\varphi(x)$ satisfy $\left(\Phi_{1}\right)$. Then for every $\eta>0(\eta \leqq 1)$ there exists $\alpha_{*}=\alpha_{*}(\eta)>0$ ((3.49) is satisfied for $|\alpha|=\alpha_{*}$ and $\alpha_{*}$ depends on $\left.\eta, b, \varphi(x)\right)$ such that if $\alpha<0,|\alpha|<\alpha_{*}$, then $s(t, \alpha)$ and its t-derivative $\dot{s}(t, \alpha)$ satisfy

$$
\limsup _{t \rightarrow \infty}\left|\frac{s(t, \alpha) \dot{s}(t, \alpha)}{|\alpha|}-1\right| \leqq \eta .
$$

Proof. For the sake of clarity we first prove the theorem in the case $b=\varphi(b)$ and under the restriction

$$
\|\dot{\varphi}\|=\underset{b<x<\infty}{\operatorname{lou} . b .}|\dot{\varphi}(x)|<\frac{1}{4} \eta .
$$

Taking $\delta=\frac{1}{4}$ in (9.5) (this determines, by the proof of Theorem 6 , the constants $H_{0}, \alpha^{*}$ such that (9.5) holds if $\left.|\alpha|<\alpha^{*}\right)$ and $\alpha_{*}$ such that

$$
2 C+\frac{b^{2}}{2}+\frac{3}{4 \delta} H_{0}^{2}\|\varphi\|^{2} \leqq \frac{b^{2}}{2 \alpha_{*}}, \quad \alpha^{*}\left(\frac{1}{4}\right)<\alpha_{*},
$$

we get

$$
s^{2}(t) \leqq 4|\alpha| t+4 b^{2} \quad \text { if } \quad|\alpha|<\alpha_{*} .
$$

In the same way we get

$$
s^{2}(t) \geqq \frac{2}{3}|\alpha| t+\frac{4}{5} b^{2} \quad \text { if } \quad|\alpha|<\alpha_{*} .
$$

The inequalities (9.10), (9.11) will play an essential role in the following. For later purposes we now introduce a positive number $t_{0}$ which satisfies the inequality

$$
b \leqq \frac{1}{16} \eta t_{0}^{\frac{1}{2}},
$$

where $\eta$ is the number which appears in the statement of the theorem. $t_{0}$ depends only on $b$ and $\eta$. Denoting

$$
M(t)=\underset{0 \leqq \tau \leqq t}{\operatorname{lu} . \mathrm{b} .}|v(\tau)|
$$

we shall prove that to every $\eta>0$ there exists $\alpha_{*}=\alpha_{*}(\eta)$ such that if $|\alpha|<\alpha_{*}$ then

$$
M(t)<\eta \quad \text { all } t<\infty .
$$

Since, by (1.9), $\alpha v=s \dot{s}+\alpha$, the proof of Theorem 7 under the restrictions made above is completed.

Proof of (9.14). We first prove (9.14) for all $t<t_{0}$. Clearly, $M(0)=$ $\left|u_{x}(b, 0)\right|=|\dot{\varphi}(b)|<\eta$ by (9.8). The set of points $t$ such that $M(t)<\eta$ is thus nonempty. Evidently it is an open set. If we prove that all its limit points $t$, 
$t \leqq t_{0}$, belong to it, then the proof of (9.14) for $t \leqq t_{0}$ is completed. Let $t \leqq t_{0}$ be such that $M(\tau)<\eta$ for $\tau<t$. We have to prove that $M(t)<\eta$, or that $|v(t)|<\eta$.

Using (2.6) we get

$$
s(t)-s(\tau) \leqq \frac{s^{2}(t)-s^{2}(\tau)}{2 b} \leqq \frac{|\alpha|}{b}(1+\eta)(t-\tau) \leqq \frac{2|\alpha|}{b}(t-\tau),
$$

since $\eta \leqq 1$. Replacing the first two terms on the right side of (2.5) by (4.1) and using (9.15), we get

$$
|v(t)| \leqq 2\|\dot{\varphi}\|+\frac{2|\alpha|}{b} t_{0}^{\frac{1}{2}}+\frac{2|\alpha|}{b} \eta t_{0}^{\frac{1}{2}}+\frac{2|\alpha|}{b} \eta t_{0}^{\frac{1}{2}} .
$$

Taking $\alpha_{*}$ to satisfy

$$
\frac{2 \alpha_{*}}{b} t_{0}^{\frac{1}{2}}<\frac{1}{4} \eta, \quad \frac{4 \alpha_{*}}{b} t_{0}^{\frac{1}{2}}<\frac{1}{4}
$$

and using (9.8), we conclude from (9.16) that $|v(t)|<\eta$.

Having proved (9.14) for all $t \leqq t_{0}$ we proceed to prove it for $t>t_{0}$. We shall need to use the inequality (9.22) below. To prove this inequality, we first use Green's formula with the functions 1 and $K(x, t ; \xi, \tau)$ in the domain $s(\tau)<\xi<\infty$, $0<\tau<t$ and thus derive the identity

$$
\begin{aligned}
1=\int_{b}^{\infty} K(x, t ; \xi, 0) d \xi-\int_{0}^{t} K_{x}(x, t ; s(\tau), \tau) d \tau & \\
& -\int_{0}^{t} K(x, t ; s(\tau), \tau) \dot{s}(\tau) d \tau .
\end{aligned}
$$

Taking $x \rightarrow s(t)+0$ and using Lemma 1 of Part I, we obtain

$$
\begin{aligned}
-\int_{0}^{t} K_{x}(s(t), t ; s(\tau), \tau) d \tau-\int_{0}^{t} K(s(t), t ; s(\tau), \tau) \dot{s}(\tau) d \tau \\
=\frac{1}{2}-\int_{b}^{\infty} K(s(t), t ; \xi, 0) d \xi \equiv I
\end{aligned}
$$

Using (9.10) we get

$$
|I|=\int_{b}^{s(t)} K(s(t), t ; \xi, 0) d \xi \leqq \frac{s(t)-b}{2(\pi t)^{\frac{1}{2}}} \leqq|\alpha|^{\frac{1}{2}}+\frac{b}{t^{\frac{1}{2}}}
$$

Taking $\alpha_{*}$ to satisfy

$$
16\left(\alpha_{*}\right)^{\frac{1}{2}}<\eta
$$

and using (9.12), we conclude from (9.20) that $|I|<\frac{1}{8} \eta$. Substituting this in (9.19) we obtain

$$
\left|\int_{0}^{t} K_{x}(s(t), t ; s(\tau), \tau) d \tau+\int_{0}^{t} K(s(t), t ; s(\tau), \tau) \dot{s}(\tau) d \tau\right|<\frac{1}{8} \eta
$$


The last inequality is decisive in the estimation of $M(t)$ for $t>t_{0}$.

In view of the previous estimates of $M(t)$, the proof of (9.14) is equivalent to the following statement: If $M(\tau)<\eta$ for all $\tau<t$ where $t$ is any number larger than $t_{0}$, then $|v(t)|<\eta$. To prove the last statement we replace the first two terms on the right side of (2.5) by (4.1) and make use of (1.9), (9.8) and (9.22). We obtain

$$
\begin{array}{rl}
|v(t)| \leqq 2\|\dot{\varphi}\|+\mid 2 \int_{0}^{t} & K(s(t), t ; s(\tau), \tau) \dot{s}(\tau) d \tau \\
& +2 \int_{0}^{t} K_{x}(s(t), t ; s(\tau), \tau) v(\tau) d \tau \mid \\
\leqq & \frac{3}{4} \eta+\left|2 \int_{0}^{t} K_{x}(s(t), t ; s(\tau), \tau)[1-v(\tau)] d \tau\right|
\end{array}
$$

It remains to estimate the last integral. Using (2.6) and (9.11) we get

$$
\frac{s(t)-s(\tau)}{t-\tau} \leqq \frac{s^{2}(t)-s^{2}(\tau)}{s(t)(t-\tau)} \leqq \frac{4|\alpha|[1+M(t)]}{(|\alpha| t)^{\frac{1}{2}}} \leqq \frac{8|\alpha|^{\frac{1}{2}}}{t^{\frac{3}{2}}} .
$$

Using (9.24), we find that

$$
\begin{aligned}
\mid 2 \int_{0}^{t} K_{x}(s(t), t ; s(\tau), \tau) & {[1-v(\tau)] d \tau \mid } \\
\leqq & \frac{8|\alpha|^{\frac{1}{2}}}{t^{\frac{1}{2}}}[1+M(t)] \int_{0}^{t} \frac{d \tau}{2 \pi^{\frac{1}{2}}(t-\tau)^{\frac{1}{2}}} \leqq 16|\alpha|^{\frac{1}{2}} .
\end{aligned}
$$

Taking $\alpha_{*}$ to satisfy

$$
16\left(\alpha_{*}\right)^{\frac{1}{2}}<\frac{1}{4} \eta
$$

and combining (9.26), (9.25) with (9.23), we get $|v(t)|<\eta$ for $|\alpha|<\alpha_{*}, \alpha<0$, and the proof is completed in the case $b=\varphi(b)$ and under the restriction (9.8).

Completion of the proof. It ramains to prove the theorem without making the restrictions (9.8) and $b=\varphi(b)$. We shall show that the proof follows from the special case treated above, provided we prove the following statement:

For every $\eta>0$ there exists $a \sigma>0$ depending on $\eta, b, \varphi(x)$, and there exists an $\alpha_{1}$ depending on $\sigma, \eta, b, \varphi(x)$, such that

$$
\left|u_{x}(x, \sigma, \alpha)\right|<H \eta \quad \text { for } s(\sigma)<x<\infty, \quad|\alpha|<\alpha_{1},
$$

where $H$ is a universal constant.

Indeed, from the proof of Theorem 3 we have

$$
\underset{8(\sigma)<x<\infty}{\operatorname{l.u.b.}}|u(x, \sigma, \alpha)| \leqq A_{1},
$$


and from the estimates of $\S 5$ (in particular, (5.5), (5.11), (5.12)) we also have

$$
\int_{s(\sigma, \alpha)}^{\infty}|u(x, \sigma, \alpha)| d x<\frac{\epsilon}{|\alpha|}+A_{2}
$$

where $\epsilon$ is any given positive number and $A_{1}, A_{2}$ are constants independent of $\alpha$ (but they depend on $\sigma, b, \varphi(x)$ and $\epsilon$ ). Analogues of (9.5), (9.6) for the function $\tilde{s}(t) \equiv s(t+\sigma)$ are now easily derived with the aid of (9.29).

We also find, upon differentiating (2.2) with respect to $x$, that

$$
\lim _{x \rightarrow \infty}\left|u_{x}(x, \sigma, \alpha)\right|=0 \text {. }
$$

Combining (9.28)-(9.30) with (9.27), we can apply with slight modifications the method used in the previous special case, replacing $\varphi(x)$ by $u(x, \sigma, \alpha)$ and $t=0$ by $t=\sigma$.

Proof of (9.27). The first step consists in proving that for any given $\eta>0$ one can find $\sigma$ depending upon $\eta, b$ and $\varphi(x)$ (but independent of $\alpha$ ) such that

$$
t^{\frac{1}{2}}|v(t, \alpha)| \leqq \eta \sigma^{\frac{1}{2}} \text { if } t \leqq \sigma,
$$

provided $|\alpha| \leqq \alpha_{1}, \alpha_{1}$ depending upon $\sigma, \eta, b$ and $\varphi(x)$.

Choose $\sigma$ such that

$$
2(b+\|\varphi\|) \sigma^{-\frac{1}{2}}<\frac{1}{2} \eta .
$$

By the proof of Theorem 3, there exists a constant $M$, depending on $\sigma, b, \varphi(x)$ but independent of $\alpha$, such that

$$
\underset{0 \leqq t<\sigma}{\text { l.u.b. }} t^{\frac{1}{2}}|v(t, \alpha)| \leqq M .
$$

Using (2.6) and (9.33) we get

$$
\frac{|s(t, \alpha)-s(\tau, \alpha)|}{t-\tau} \leqq \frac{2|\alpha|}{b t^{\frac{1}{2}}}(M+1)
$$

Recalling that (3.1) holds with $\bar{w}=\bar{v}=t^{\frac{1}{2}} v(t)$, we obtain, on making use of (9.34),

$$
t^{\frac{1}{2}}|v(t, \alpha)| \leqq 2(b+\| \varphi||)+\frac{2|\alpha| t}{b}+\frac{2|\alpha|}{b} t^{\frac{1}{2}} M+\frac{2|\alpha|}{b} M(M+1) .
$$

Taking $\alpha_{1}$ such that it satisfies

$$
2 \alpha_{1}\left(\frac{\sigma}{b}+\frac{\sigma^{\frac{1}{2}} M}{b}+\frac{M(M+1)}{b}\right)<\frac{1}{2} \eta \sigma^{\frac{1}{2}}
$$

and using (9.32), we conclude that the right side of (9.35) is bounded by $\eta \sigma^{\frac{1}{3}}$ provided $t<\sigma,|\alpha| \leqq \alpha_{1}$. This completes the proof of (9.31).

We proceed to prove (9.27). Differentiating (2.2) with respect to $x$ and per- 
forming integration by parts (see (2.4)) we obtain, on using (1.9) and taking $t=\sigma$,

$$
\begin{aligned}
\left|u_{x}(x, \sigma, \alpha)\right| \leqq(b+\|\varphi\|) \sigma^{-\frac{1}{2}} & \\
& +\left|\int_{0}^{\sigma} K(x, \sigma ; s(\tau, \alpha), \tau) \frac{\alpha v(\tau, \alpha)-\alpha}{s(\tau, \alpha)} d \tau\right| \\
& +\left|\int_{0}^{\sigma} K_{x}(x, \sigma ; s(\tau, \alpha), \tau) v(\tau, \alpha) d \tau\right| .
\end{aligned}
$$

Using (9.31) we find that

$$
\begin{aligned}
\mid \int_{0}^{\sigma} K(x, \sigma ; s(\tau, \alpha), \tau) & \frac{\alpha v(\tau, \alpha)-\alpha}{s(\tau, \alpha)} d \tau \mid \\
& \leqq \frac{|\alpha|}{b} \eta \sigma^{\frac{1}{2}} \int_{0}^{\sigma} \frac{d \tau}{2 \pi^{\frac{1}{2}}(\sigma-\tau)^{\frac{1}{2}} \tau^{\frac{1}{2}}}+\frac{|\alpha|}{b} \int_{0}^{\sigma} \frac{d \tau}{2 \pi^{\frac{1}{2}}(t-\tau)^{\frac{1}{2}}} \\
& \leqq \frac{|\alpha|}{b} \eta \sigma^{\frac{1}{2}}+\frac{|\alpha|}{b} \sigma^{\frac{1}{2}}<\eta,
\end{aligned}
$$

provided $\alpha_{1}$ satisfies

$$
\alpha_{1} \frac{\sigma^{\frac{1}{3}}}{b}<\frac{1}{2} \eta
$$

It remains to estimate

$$
I \equiv I(x, \sigma, \alpha) \equiv \int_{0}^{\sigma} K_{x}(x, \sigma ; s(\tau, \alpha), \tau) v(\tau, \alpha) d \tau
$$

Estimation of $I$. Divide the integral into two parts

$$
I=\int_{0}^{\frac{1}{2} \sigma}+\int_{\frac{1}{2} \sigma}^{\sigma}=I_{1}+I_{2} \text {. }
$$

Using (9.31) and the inequality $x \exp \left\{-x^{2}\right\} \leqq 2^{\frac{1}{2}}$, we get

$$
\left|I_{1}\right| \leqq \int_{0}^{\frac{1}{2} \sigma} \frac{1}{4(\sigma-\tau)} \frac{\sigma^{\frac{1}{2}} \eta}{\tau^{\frac{1}{2}}} d \tau \leqq \frac{\sigma^{\frac{1}{2}} \eta}{2 \sigma} \int_{0}^{\frac{1}{2} \sigma} \frac{d \tau}{\tau^{\frac{1}{2}}}=\eta .
$$

To estimate $I_{2}$ we first note by (1.9), (9.31) that

(9.43) $\frac{s(\sigma)-s(\tau)}{\sigma-\tau}=\dot{s}(\tilde{\tau}) \leqq \frac{|\alpha|}{b}\left[1+\frac{\eta \sigma^{\frac{1}{2}}}{\left(\frac{1}{2} \sigma\right)^{\frac{1}{2}}}\right] \leqq \frac{3|\alpha|}{b}$ provided $\quad \frac{1}{2} \sigma<\tau<\sigma$.

Here $\tau<\tilde{\tau}<\sigma$. With the aid of some inequalities which appear in the proof of Lemma 1 of Part I with $t=\sigma, t-\delta=\frac{1}{2} \sigma$, and on using (9.43), we obtain, after some elementary calculation,

$$
\int_{\frac{1}{2} \sigma}^{\sigma}\left|K_{x}(x, \sigma ; s(\tau, \alpha), \tau)\right| d \tau \leqq H_{1}\left[1+\frac{|\alpha| \sigma^{\frac{1}{2}}}{b}+\frac{|\alpha|^{2} \sigma}{b^{2}}\right]
$$


where $H_{1} \geqq 1$ is a universal constant. Substituting (9.31) into $I_{2}$ and using (9.44) we obtain

$$
\left|I_{2}\right| \leqq 2 H_{1} \frac{\sigma^{\frac{1}{2}} \eta}{\left(\frac{1}{2} \sigma\right)^{\frac{1}{2}}} \leqq 4 H_{1} \eta,
$$

since, by (9.39), $\alpha_{1}$ satisfies

$$
\frac{\alpha_{1} \sigma^{\frac{1}{2}}}{b}<\frac{1}{2}
$$

Combining (9.45), (9.42) with (9.41) we get

$$
|I| \leqq\left(4 H_{1}+1\right) \eta \text {. }
$$

Combining (9.40), (9.47), (9.32), (9.38) with (9.37), the proof of (9.27) is completed.

9.3. Behavior of $u(x, t, \alpha)$ as $t \rightarrow \infty$. Using the previous results we proceed in this section to study the behavior of $u(x, t, \alpha)$ for small $|\alpha|$ as $t \rightarrow \infty$. Denote for brevity

$$
\lambda=(2|\alpha|)^{\frac{1}{2}} .
$$

It is enough to consider the case $\varphi(b)=b$, since in the case $\varphi(b) \neq b$ we can start the whole argument with $t=\sigma$ instead of $t=0$.

By (9.5), (9.6) we have, for $0<t<\infty$,

$$
s(t, \alpha)-\lambda t^{\frac{1}{2}}=t^{\frac{1}{2}} o(\lambda, t)+A \quad(A>0)
$$

where $A$ and $1 / A$ are appropriate functions bounded by constants independent of $\lambda, t$, and where $o(\lambda, t)$ is a function of $(\lambda, t)$ such that $\lambda^{-1} o(\lambda, t) \rightarrow 0$ as $\lambda \rightarrow 0$, uniformly with respect to $t, 0<t<\infty$. In what follows we shall denote by $o(\lambda, t)$ (or $o(\lambda, x, t)$ ) any function of $(\lambda, t)$ (or $(\lambda, x, t)$ ) which satisfies $\lambda^{-1} o(\lambda, t) \rightarrow 0$ (or $\lambda^{-1} o(\lambda, x, t) \rightarrow 0$ ) as $\lambda \rightarrow \infty$, uniformly with respect to $t$ (or $(x, t)$ ).

From the proof of Theorem 7 it follows that for every $\eta>0$ we can choose $\alpha_{*}>0$ such that if $|\alpha|<\alpha_{*}$,

$$
|v(t, \alpha)|=\left|\frac{s(t, \alpha) \dot{s}(t, \alpha)}{|\alpha|}-1\right|<\eta \text { for all } t \geqq \sigma
$$

where $\sigma=\sigma(\eta)$ depends only on $b, \varphi(x), \eta$ (see (9.32)) and $\alpha_{*}$ depends on $b$, $\varphi(x), \eta$ and $\sigma$. Taking, in particular, $\eta=1$ we get

$$
|v(t, \alpha)|<1 \text { for all } t \geqq \sigma_{0} \quad\left(\sigma_{0}=\sigma(1)\right)
$$

provided $|\alpha|<\alpha_{*}$.

Now, by (9.49) we have

$$
\begin{array}{r}
\frac{|\alpha|}{s(t, \alpha)}=\frac{|\alpha|}{t^{\frac{1}{2}}[\lambda+o(\lambda, t)]+A}=\frac{|\alpha|}{t^{\frac{1}{2}}[\lambda+o(\lambda, t)]}\left[1+o\left(\frac{|A|}{\lambda t^{\frac{1}{2}}}\right)\right] \\
=\frac{\lambda}{2 t^{\frac{1}{2}}\left[1+\lambda^{-1} o(\lambda, t)\right]}+\frac{\text { bounded function }}{t}
\end{array}
$$


where $O(y)$ is a function satisfying $|O(y)| \leqq$ const. $|y|$, provided $\lambda t^{\frac{1}{2}} \geqq K$ where $K$ is a sufficiently large constant. Take $\lambda$ to be such that the inequality $\lambda[\sigma(\eta)]^{\frac{1}{2}}<K$ holds, where $\sigma=\sigma(\eta)$ is such that (9.50) holds for all $t>\sigma(\eta)$. Then $\lambda t^{\frac{1}{2}} \geqq K$ implies $t \geqq \sigma(\eta)$, and hence if $\lambda t^{\frac{1}{2}} \geqq K$ then (9.50) holds. Since (9.52) also holds, we obtain

$$
\dot{s}(t, \alpha)-\frac{\lambda}{2 t^{\frac{1}{2}}}=\frac{1}{2 t^{\frac{1}{2}}} \tilde{o}(\lambda, t)+\frac{B}{t} \quad \text { if } \quad \lambda t^{\frac{1}{3}} \geqq K
$$

where $B$ is an appropriate function bounded by constants depending only on $b, \varphi(x)$, and $\tilde{o}(\lambda, t)$ satisfies

$$
\lambda^{-1} \tilde{o}(\lambda, t) \rightarrow 0 \text { as } \lambda \rightarrow 0 \text {, uniformly in } t \text { such that } \lambda t^{\frac{1}{2}} \geqq K .
$$

We proceed to prove (9.53) for $\lambda t^{\frac{1}{2}} \leqq K$ and with $\tilde{o}(\lambda, t) \equiv 0$. If $t \geqq \sigma_{0}$, then, using (9.51) and (9.48), we obtain

$$
|\dot{s}|=\left|\frac{s \dot{s}}{\alpha}\right| \frac{|\alpha|}{s}=|v-1| \frac{|\alpha|}{s} \leqq \frac{\lambda^{2}}{b} \leqq \frac{K^{2}}{b t} \quad \text { if } \quad \lambda t^{\frac{1}{2}} \leqq K .
$$

For $t<\sigma_{0}$ we have (see the proof of Theorem 3)

$$
|v(t, \alpha)| \leqq K_{1}
$$

where $K_{1}$ is a constant independent of $\alpha$. We conclude, in the same manner as before, that

$$
|\dot{s}|=|v-1| \frac{|\alpha|}{s} \leqq \frac{1+K_{1}}{2} \frac{\lambda^{2}}{b} \leqq \frac{1+K_{1}}{2} \frac{K^{2}}{b t} .
$$

Combining (9.55), (9.56) with the inequality

$$
\frac{\lambda}{2 t^{\frac{1}{2}}} \leqq \frac{K}{2 t}, \text { provided } \lambda t^{\frac{1}{2}} \leqq K,
$$

we conclude that if $\lambda t^{\frac{3}{3}} \leqq K$ then

$$
\left|\dot{s}(t, \alpha)-\frac{\lambda}{2 t^{\frac{1}{2}}}\right| \leqq \frac{\text { const. }}{t},
$$

and the proof of (9.53) for $\lambda t^{\frac{1}{2}} \leqq K$ and with $\tilde{o}(\lambda, t) \equiv 0$ is completed. Combining this result with (9.53), recalling the property (9.54) of $\tilde{o}(\lambda, t)$, and using the notation of $o(\lambda, t)$ introduced above, we conclude that

$$
\dot{s}(t, \alpha)-\frac{\lambda}{2 t^{\frac{1}{2}}}=\frac{1}{2 t^{\frac{1}{3}}} o(\lambda, t)+\frac{B}{t} \quad(0<t<\infty)
$$

where $B$ is a function bounded by a constant depending only on $b, \varphi(x)$.

Using (9.57), (9.52) we find that the function

$$
\tilde{u}(x, t, \alpha)=u\left(x-\lambda t^{\frac{1}{2}}+s(t, \alpha), t, \alpha\right)-\left[s(t, \alpha)-\lambda t^{\frac{1}{2}}\right]
$$


satisfies the equation

$$
\tilde{u}_{x x}-\tilde{u}_{t}=\frac{o(\lambda, x, t)}{t^{\frac{3}{2}}}+\frac{\widetilde{B}}{t}, \quad|\widetilde{B}| \leqq K_{2},
$$

where $K_{2}$ depends only on $b$ and $\varphi(x)$. Here we used the fact that $u_{x}(x, t, \alpha)$ is a bounded function for $s(t, \alpha)<x<\infty, 0<t<\infty$ and $|\alpha|$ sufficiently small. The proof of this fact follows by using the maximum principle for $u_{x}$, noting that, on the boundary $x=s(t, \alpha), u_{x}(s(t, \alpha), t, \alpha)=v(t, \alpha)$ and $v(t, \alpha)$ is a bounded function for $0<t<\infty$ and $|\alpha|$ sufficiently small (by (9.51) and Theorem 3), and noting that, on the boundary $t=0, u_{x}(x, 0)=\dot{\varphi}(x)$ is a bounded function.

The function $\tilde{u}(x, t, \alpha)$ satisfies the boundary conditions

$$
\tilde{u}\left(\lambda t^{\frac{1}{2}}, t, \alpha\right)=\lambda t^{\frac{1}{2}}, \quad \tilde{u}(x, 0, \alpha)=\varphi(x+b)-b \equiv \tilde{\varphi}(x) .
$$

The function

$$
\tilde{U}(x, t, \alpha)=\frac{\tilde{u}(x, t, \alpha)}{x}
$$

satisfies the system

$$
\begin{aligned}
& \tilde{U}_{x x}+\frac{2}{x} \tilde{U}_{x}-\tilde{U}_{t}=\frac{o(\lambda, x, t)}{x t^{\frac{3}{2}}}+\frac{\widetilde{B}}{x t}, \\
& \tilde{U}\left(\lambda t^{\frac{3}{2}}, t, \alpha\right)=1 \\
& \tilde{U}(x, 0, \alpha)=\frac{\tilde{\varphi}(x)}{x} .
\end{aligned}
$$

We try to find a function

$$
V(x, t, \alpha)=f(z, \lambda) \quad \text { where } \quad z=\frac{x}{t^{\frac{1}{2}}}
$$

which satisfies the system

$$
\begin{aligned}
& V_{x x}+\frac{2}{x} V_{x}-V_{t}=0, \\
& V\left(\lambda t^{\frac{1}{2}}, t, \alpha\right)=1, \\
& V(x, 0, \alpha)=0 .
\end{aligned}
$$

We obtain for $f(z, \lambda)$ the system

$$
\begin{gathered}
f^{\prime \prime}+\left(\frac{2}{z}+\frac{z}{2}\right) f^{\prime}=0 \quad\left(f^{\prime}=\frac{\partial f}{\partial z}\right), \\
f(\lambda, \lambda)=1, \quad f(\infty, \lambda)=0 .
\end{gathered}
$$

The solution is easily found to be

$$
f(z, \lambda)+C(\lambda) \int_{z}^{\infty} \frac{1}{\zeta^{2}} \exp \left\{-\frac{\zeta^{2}}{4}\right\} d \zeta
$$


where

$$
C(\lambda)=\left[\int_{\lambda}^{\infty} \frac{1}{\zeta^{2}} \exp \left\{-\frac{\zeta^{2}}{4}\right\} d \zeta\right]^{-1}
$$

Defining

$$
W=\tilde{U}-V, \quad w=x W
$$

we proceed to estimate $w$. $w$ satisfies the system

$$
\begin{gathered}
w_{x x}-w_{t}=\frac{o(\lambda, x, t)}{t^{\frac{1}{2}}}+\frac{\widetilde{B}}{t}, \quad|\widetilde{B}| \leqq K_{2}, \\
w\left(\lambda t^{\frac{1}{2}}, t, \alpha\right)=0, \\
w(x, 0, \alpha)=\tilde{\varphi}(x) \equiv \varphi(x+b)-b .
\end{gathered}
$$

It is also easy to verify, on using the definition of $w$, that $w, w_{x}$ remain bounded as $x \rightarrow \infty$, uniformly with respect to $t$ in bounded intervals. The nonhomogeneous term in (9.64) has a singularity for $t=0$ which might cause complications in trying to estimate $w$. Hence we consider the differential equation (9.64) only in the domain $1<t<\infty, s(t)<x<\infty$. Equation (9.66) is then replaced by

$$
\begin{aligned}
w(x, 1, \alpha)=u(x+s(1, \alpha)-\lambda, & 1, \alpha) \\
& -[s(1, \alpha)-\lambda]-x f(x, \lambda) \equiv \tilde{\varphi}(x, \alpha) .
\end{aligned}
$$

Using the boundedness of $s(1, \alpha)$ with respect to $\alpha$ and the explicit form of $f$ in (9.61), (9.62), we conclude that

$$
|\tilde{\varphi}(x, \alpha)| \leqq K_{3} \quad\left(K_{3} \quad \text { independent of } \alpha\right) .
$$

Consider the function

$$
F(x, t)=2|o(\lambda)| t^{\frac{1}{3}}+K_{2} \log t+K_{3}, \quad|o(\lambda)|=\underset{\substack{1<t<\infty \\ s(t)<x<\infty}}{1 . u . b}|o(\lambda, x, t)|,
$$

where $o(\lambda, x, t)$ is precisely the function which appears in (9.64). $F$ satisfies the system

$$
\begin{gathered}
F_{x x}-F_{t}=-\frac{|o(\lambda)|}{t^{\frac{1}{2}}}-\frac{K_{2}}{t} \text { for } 1<t<\infty, \quad s(t)<x<\infty, \\
F\left(\lambda t^{\frac{1}{2}}, t\right) \geqq 0 \text { for } 1<t<\infty, \\
F(x, 1) \geqq K_{3} \text { for } s(1)<x<\infty .
\end{gathered}
$$

We shall now prove that $w \leqq F$. We first notice that $F, F_{x}$ remain bounded as $x \rightarrow \infty$, uniformly with respect to $t$ in finite intervals. Hence the same is true of

$$
v(x, t) \equiv v=F-w .
$$

Define $\tilde{v}(y, t)=v(x, t)$ where $y=x-\lambda t^{\frac{1}{2}}$. Then $\tilde{v}$ satisfies the system

$$
\tilde{v}_{y y}-\tilde{v}_{t}+\frac{\lambda}{2 t^{\frac{1}{2}}} \tilde{v}_{y} \leqq 0 \text { for } 1<t<\infty, \quad 0<y<\infty,
$$




$$
\begin{aligned}
& \tilde{v}(0, t) \geqq 0 \quad \text { for } \quad 1<t<\infty, \\
& \tilde{v}(y, 1) \geqq 0 \quad \text { for } \quad 0<y<\infty .
\end{aligned}
$$

Define

$$
b(y, t)=\left\{\begin{array}{ccc}
\frac{\lambda}{2 t^{\frac{1}{2}}} & \text { if } & 0 \leqq y<\infty \\
-\frac{\lambda}{2 t^{\frac{1}{2}}} & \text { if } & -\infty<y<0
\end{array}\right.
$$

and let $\Gamma(y, t ; \eta, \tau)$ be the fundamental solution of the equation $z_{y y}-z_{t}+$ $b(y, t) z_{y}=0$ in the strip $\mid<t<\infty$. It can be constructed, for instance, by the method of [1]. It can also be shown that

$$
\Delta(y, t ; \eta, \tau)=\Gamma(y, t ; \eta, \tau)-\Gamma(-y, t ; \eta, \tau)
$$

is Green's function of the equation $z_{y y}-z_{t}+\lambda z_{y} / 2 t^{\frac{1}{2}}=0$ in the domain $1<t<\infty, 0<y<\infty$. As a function of $(y, t)$ it satisfies this equation; and as a function of $(\eta, \tau)$ it satisfies the adjoint equation. It also vanishes for $y=0$.

Using Green's identity with the functions $\widetilde{v}(\eta, \tau), \Delta(y, t ; \eta, \tau)$ in the domain $1<\tau<t-\epsilon, 0<\eta<k$, then letting $\epsilon \rightarrow 0, k \rightarrow \infty$ and using the boundedness of $\tilde{v}(k, \tau), \tilde{v}_{\eta}(k, \tau)$ as $k \rightarrow \infty$, we obtain an integral representation for $\tilde{v}(y, t)$ in terms of its boundary values $v(y, 1), v(0, t)$ and the left side of (9.71). Using (9.71), (9.72), (9.73), we then conclude that $\tilde{v}(y, t) \geqq 0$. Hence $v(x, t) \geqq 0$. Using (9.69), (9.70), we conclude

$$
w(x, t, \alpha) \leqq 2|o(\lambda)| t^{\frac{1}{2}}+K_{2} \log t+K_{3} .
$$

In a similar way we obtain $F+w \geqq 0$. Hence

$$
-w(x, t, \alpha) \leqq 2|o(\lambda)| t^{\frac{1}{2}}+K_{2} \log t+K_{3} .
$$

Combining the inequalities (9.74), (9.75) and using the definitions of $w, W$, $V, U, u$, we get

$$
\left|\tilde{u}(x, t, \alpha)-x f\left(\frac{x}{t^{\frac{1}{2}}}, \lambda\right)\right| \leqq 2|o(\lambda)| t^{\frac{1}{2}}+K_{2} \log t+K_{3} .
$$

Using the definition of $\tilde{u}$ in (9.58) and (9.49) we obtain

$$
\left|u(x, t, \alpha)-x f\left(\frac{x}{t^{\frac{1}{2}}}+o(\lambda), \lambda\right)\right| \leqq|o(\lambda)| t^{\frac{1}{2}}+K_{2} \log t+K_{3}
$$

where $o(\lambda)$ is now used to denote any appropriate function of $\lambda$ which satisfies $\lambda^{-1} o(\lambda) \rightarrow 0$ as $\lambda \rightarrow 0$.

Formula (9.77) is the desired approximation formula to the solution $u(x, t, \alpha)$. We note that one is mainly interested in evaluating $u(x, t, \alpha)$ in the neighborhood of the surface of the drop, since at far points the initial density of the vapor is unaffected by the process of condensation. Since, as was previously proved, 
the radius of the drop grows like $\lambda t^{\frac{1}{2}}$, we are interested in evaluating $u(x, t, \alpha)$ at points

$$
(x, t)=\left(Q \lambda t^{\frac{1}{2}}, t\right) \text { where } 0<Q<\text { const. }
$$

Since

$$
\int_{\mu}^{\infty} \frac{1}{\zeta^{2}} \exp \left\{-\frac{\zeta^{2}}{4}\right\} d \zeta=\frac{1}{\mu} \exp \left\{-\frac{\mu^{2}}{4}\right\}-\frac{1}{2} \int_{\mu}^{\infty} \exp \left\{-\frac{\zeta^{2}}{4}\right\} d \zeta
$$

we find that at points of the form (9.78)

$$
x f\left(\frac{x}{t^{\frac{1}{2}}}\right) \text { is approximately } \frac{\lambda t^{\frac{1}{2}}}{Q},
$$

so that the right side of (9.77) indeed represents an error term for $\lambda$ small and $t$ large.

Substituting (9.79) into (9.77) and using the notations of $\S 1$, we derive, after omitting terms of small order of magnitude, the following formula:

$$
c(x, t)=c_{0}-\frac{1}{Q}\left(c_{0}-g\right) .
$$

This is an approximate law which becomes more precise as $\lambda$ becomes smaller and as $t$ becomes larger, $Q$ being bounded. It states that the density at any point $P$ of the supersaturated vapor is a linear function of the ratio of the radius of the drop to the distance of $P$ from the drop's center.

\section{ReFERENCES}

[1] F. G. Dressel, The fundamental solution of the parabolic equation II, Duke Math J., 13 (1946), pp. 61-70.

[2] A. Friedman, Free boundary problems for parabolic equations I: Melting of Solids, J. Math. and Mech., 8 (1959), pp. 499-518.

[3] J. B. Keller, I. I. Kolodner \& P. D. Ritger, Decay of drop by evaporation and growth by condensation, IMM-NYU, 1952, No. 183.

[4] I. I. Kolodner, Decay of drops by evaporation, $I M M-N Y U$, 1953, No. 199.

[5] I. I. KolodNer, Growth of drops by condensation, $I M M-N Y U, 1955$, No. 215.

[6] I. I. Kolodner, Free boundary problems for the heat equation with applications to problems of change of phase, Comm. Pure Appl. Math., 9 (1956), pp. 1-31.

[7] I. I. Kolodner \& P. D. Ritger, Evaporation of a collection of liquid drops, IMMNYU, 1954, No. 213.

[8] L. Nirenberg, A strong maximum principle for parabolic equations, Comm. Pure Appl. Math., 6 (1953), pp. 167-177.

[9] W. Pogorzelski, Etude de la solution fundamentale de l'équation parabolique, Ricerche di Mat., 5 (1956), pp. 25-57.

[10] W. Pogonzelskr, Propriétés des intégrales de l'équation parabolique normale, Ann. Polon. Math., 4 (1957), pp. 62-92.

University of California

Berkeley, California

and

Indiana University

Bloomington, Indiana 